\title{
ODONTOMETRÍA Y VARIANTES MORFOLÓGICAS DENTALES EN PIEZAS DENTARIAS DEL MAYA PREHISPÁNICO DEL MUSEO NACIONAL DE ARQUEOLOGÍA Y ETNOLOGÍA DE GUATEMALA (MUNAE)
}

\author{
DENTISTRY AND DENTAL MORPHOLOGICAL VARIANTS IN PRE-HISPANIC MAYA \\ TEETH PIECES OF THE NATIONAL MUSEUM OF ARCHEOLOGY AND ETHNOLOGY OF \\ GUATEMALA (MUNAE)
}

Referencia del artículo

Fernández, F., Rosales, G. y Zuleta, S. (2019). Odontometría y variantes morfológicas dentales en piezas dentarias del maya prehispánico del museo nacional de arqueología y etnología de Guatemala (MUNAE). Revista Científica del SEP. 2(1), 11-17. DOI: https://doi.org/10.36958/sep.v2i01.22

\author{
Dr. Francisco René Fernández Rodas \\ francisco.fernandez.rodas@gmail.com \\ Dr. Guillermo Rosales Escribá \\ escribarosaleswilly@gmail.com \\ Dr. Sergio José Zuleta Mejía \\ sergio09zuleta@yahoo.com
}

\author{
Especialización en Odontología Forense con Énfasis en \\ Identificación Humana \\ Facultad de Odontología \\ Departamento de Postgrado \\ Universidad de San Carlos de Guatemala
}

Fecha de recepción: 11/07/2019

Fecha de aceptación: 25/10/2019

\section{RESUMEN \\ El propósito de este estudio fue recopilar los datos sobre la odontometría y las variantes morfológicas en la dentición permanente de los mayas prehispánicos. En la presente investigación fueron estudiadas, analizadas y medidas todas las piezas dentales permanentes evaluables (apico- coronalmente), así como las variantes morfológicas tales como: diente en pala, enanismo radicular, tubérculo de carabelli, tubérculo sextum, etc., que se encontraron en la población maya estudiada, dientes resguardados en el Museo Nacional de Arqueología y Etnología de Guatemala (MUNAE).}

Las piezas usadas para la evaluación de variantes morfológicas en el Museo Nacional de Arqueología y Etnología de Guatemala fueron un total de 529 piezas dentales, se utilizaron piezas sueltas y piezas en cráneos que cumplieran los requisitos de inclusión.

La pieza dental que presentó la mayor medida en el largo total del estudio del maya prehispánico fue el canino superior con $27.03 \mathrm{~mm}$. La pieza dental que presentó la menor medida en el largo total del estudio del maya prehispánico fue el segundo molar inferior con $19.95 \mathrm{~mm}$. Los incisivos superiores tanto centrales como laterales presentaron únicamente el tipo V. En caninos superiores la variante más frecuente de cara lingual fue el tipo III. En los primeros molares superiores la variante de cara oclusal más frecuente fue el tipo I con un $90 \%$. Un dato interesante de hacer mención es que se encontraron tres segundos premolares inferiores que presentaron la variante Con Cresta y Sin surco, esta variante es más frecuente de encontrarse en primeros premolares inferiores. En segundos molares inferiores el tipo más frecuente fue el tipo II con un $86.48 \%$.

\section{ABSTRACT}

The purpose of this study was to collect data on odontometry and morphological variants in the permanent dentition of the pre-Hispanic Maya. In the present investigation, all evaluable permanent teeth (apico-coronally) were studied, analyzed and measured, as well as morphological variants such as: shovel tooth, root dwarfism, carabelli tuber, sextum tuber, etc., which were found in the Mayan population studied, teeth protected in the National Museum of Archeology and Ethnology of Guatemala (MUNAE).

The pieces used for the evaluation of morphological variants in the National Museum of Archeology and Ethnology of Guatemala were a total of 529 teeth, loose pieces and pieces in skulls that met the inclusion requirements were used.

The tooth that presented the greatest measurement in the total length of the pre-Hispanic Maya study was the upper canine with $27.03 \mathrm{~mm}$. The tooth that presented the smallest measurement in the total length of the pre-Hispanic Maya study was the second lower molar with $19.95 \mathrm{~mm}$. Both the central and lateral upper incisors presented only type V. In upper canines, the most frequent variant of the lingual aspect was type III. In the upper first molars, the most frequent occlusal variant was type I with $90 \%$. An interesting fact to mention is that three lower second premolars were found that presented the variant With Crest and Without groove, this variant is more frequent to be found in lower first premolars. In lower second molars, the most frequent type was type II with $86.48 \%$.

As future prospects, it would be advisable to carry out this same study radiographically to determine the pre-Hispanic 
Como perspectivas de futuro sería recomendable realizar este mismo estudio radiográficamente para determinar la anatomía y morfología pulpar prehispánica, así como realizar el mismo estudio en el guatemalteco actual.

\section{PALABRAS CLAVE}

Anatomía y Morfología Dental, Odontometría, Variantes Morfológicas Dentales, Odontología del Maya Prehispánico

\section{INTRODUCCIÓN}

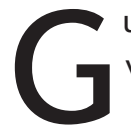
uatemala es conocida mundialmente por sus volcanes, lagos, flora y fauna, por sus colores, su cultura, sus tradiciones y por su gente amable y trabajadora; además de todo eso Guatemala tiene la dicha de ser llamada “La Cuna de la Civilización Maya”. Por todo lo descrito anteriormente, nace la inquietud de realizar un estudio que reúna por primera vez datos sobre variantes morfológicas y odontométricas de los dientes de la población maya del período prehispánico.

En la presente investigación fueron estudiadas, analizadas y medidas todas las piezas dentales permanentes evaluables (apico-coronalmente), así como las variantes morfológicas tales como: diente en pala, enanismo radicular, tubérculo de carabelli, tubérculo sextum, etc., que se encontraron en la población maya estudiada.

Para la elaboración de esta investigación se contó con el apoyo profesional del Dr. Guillermo Rosales Escribá, quien con su experiencia en el campo de la Morfología y Anatomía Dental aportó su conocimiento para poder completar este estudio, así como la colaboración de las autoridades y personal del "Museo Nacional de Arqueología y Etnología de Guatemala (MUNAE)", quienes prestaron las piezas dentales que se utilizaron para esta investigación.

El MUNAE es una institución pública guatemalteca encargada de la conservación, investigación y difusión de los bienes arqueológicos y etnológicos, pertenecientes al Patrimonio Cultural de la Nación de Guatemala.

\section{OBJETIVOS}

- Recopilar los datos sobre la odontometría y las variantes morfológicas en la dentición permanente de los mayas prehispánicos. pulp anatomy and morphology, as well as to carry out the same study in the current Guatemalan.

\section{KEYWORDS}

Dental Anatomy and Morphology, Odontometry, Dental Morphological Variants, Pre-Hispanic Maya Dentistry

- Analizar las dimensiones de las piezas dentarias del maya prehispánico de manera longitudinal, mesio-distal, buco-lingual, cervico-oclusal/ incisal, así como también las variantes morfológicas de las piezas presentes y determinar si existe relación entre estos parámetros y los encontrados en estudios actuales del ser humano.

- Poder tener una guía o documento de consulta con datos que coincidan con las características de nuestra población.

- Determinar las características morfológicas de los dientes anteriores superiores e inferiores permanentes.

- Determinar las características morfológicas de los premolares permanentes superiores e inferiores.

- Determinar las características morfológicas de los molares permanentes superiores e inferiores.

- Determinar la uni o bilateralidad de los tubérculos de Carabelli, Sextum, Selenka, Reborde Transversal Anterior (RTA) y Premolar de Leong.

\section{JUSTIFICACIÓN}

Actualmente no se cuentan con documentos que se refieran a características propias de nuestra cultura en lo que concierne a parámetros odontométricos y morfológicos, por lo cual se tomó la decisión de compilar datos de piezas de mayas prehispánicos encontradas en las diferentes regiones del país facilitadas por el Museo Nacional de Arqueología y Etnología de Guatemala 
El presente estudio sirvió para enriquecer los conocimientos de la odontometría y la morfología dental del guatemalteco y podrá ser usado como base en estudios posteriores.

\section{METODOLOGÍA}

\subsection{Variables del Estudio}

\section{a. Odontometría:}

Es el método de identificación que se basa en la obtención de medidas de uno o varios dientes que se hallan a disposición, además, se aplica para el estudio de los parámetros morfométricos de los dientes. Es la determinación correcta de la longitud de un diente.

\section{b. Dentición permanente:}

También conocida como segunda dentición. Es la que dura más tiempo y está compuesta por treinta y dos piezas dentarias, repartidas en dos grupos: dientes anteriores que son los incisivos centrales, los incisivos laterales y los caninos y dientes posteriores que son los premolares y molares. Estas piezas dentarias hacen su aparición en la cavidad bucal entre los seis y los veintiún años de edad.

\subsection{Características o variables morfológicas dentales:}

Son aquellos fenómenos que se presentan como una desviación o alteración de mayor o menor grado, no alterando esencialmente la forma macroscópica del diente, pero sí sus características generales lo suficiente para individualizarlo.

Para el presente estudio se utilizaron dos tipos de variables; las variables cuantitativas que ayudaron a examinar la odontometría y las variables cualitativas que se usaron en el análisis de variantes morfológicas.

Se realizó la odontrometría de las piezas dentales, tanto anteriores como posteriores permanentes, agrupándolas y clasificándolas por grupos anatómicos (incisivos, caninos, premolares, molares tanto superiores como inferiores a excepción de los terceros molares), a través del sistema F.D.I., tomando como base cuatro medidas: el largo total de la pieza (oclusal/ incisal- apical), las distancias mesio-distal, buco-lingual y cervico-oclusal/incisal.

Los datos obtenidos de las mediciones, fueron recolectados en las Fichas Odontométricas para la Recolección de Datos en Piezas Individuales (figura 1), las cuales fueron de utilidad para el posterior análisis estadístico de las diferentes dimensiones antes mencionadas.

Las operaciones estadísticas utilizadas en la presente investigación por recomendación del Estadista fueron: la media aritmética y la desviación estándar.

a. Media aritmética:

Se le conoce también como promedio o media, es una medida de tendencia central y es el resultado de la división de la sumatoria de todas las medidas de las piezas según su clasificación anatómica entre la cantidad de las piezas medidas.

$$
\bar{x}=\frac{x_{1}+x_{2}+x_{3}+\cdots+x_{n}}{n}
$$

$n$

b. Desviación estándar:

Es una medida de variabilidad, indica cuanta variabilidad o diferencia existe en una muestra y se representa en un porcentaje que se llama coeficiente de variación. Es la raíz cuadrada de la varianza, es una de las medidas de dispersión que es indicativa de como los valores individuales pueden diferir de la media.

La desviación estándar mide cuanto se separan los datos, su fórmula es:

$$
\mathrm{DE}=\sqrt{\frac{\sum|x-\mu|^{2}}{N}}
$$

Donde $\sum$ significa "suma de", $\mathrm{x}$ es un valor de un conjunto de datos, es la media del conjunto de datos y $\mathrm{N}$ es el número de datos.

En el proceso de las variantes morfológicas, como se mencionó anteriormente fueron usadas variables cualitativas, por lo que se empleó el porcentaje para dar a conocer el o las características morfológicas más frecuentes.

Para este estudio fue utilizada por primera vez en una investigación la "Ficha Odontológica de Variantes Morfológicas” (figura 2), diseñada por el Dr. Guillermo Rosales Escribá y modificada por la Dra. Ingrid Robles de Zuleta, debido a que ésta reúne las características puntuales para un estudio de este tipo; esta ficha cuenta con 3 páginas, el esquema sencillo pero completo de esta ficha fue la base para poder obtener las variantes morfológicas de una manera ordenada, ya que está diseñada para describir con precisión la morfología dental de cada una de las piezas de la dentición permanente. 
Figura 1

Ficha utilizada para odontometrias

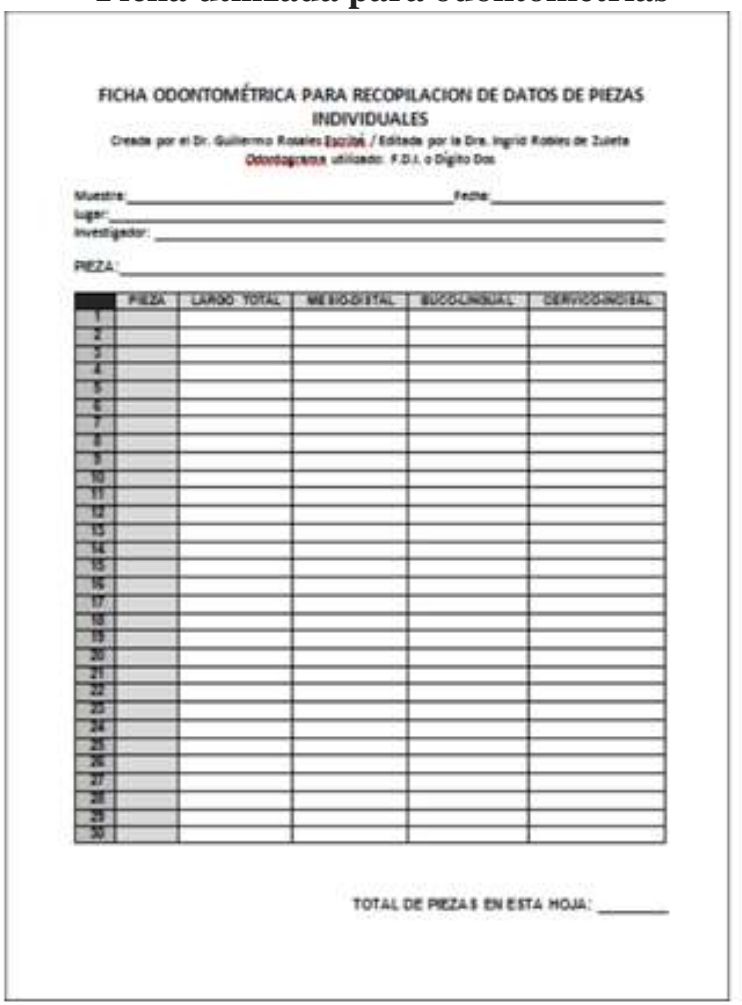

Fuente: Dr. Guillermo Rosales Escribá

Figura 2

Ficha antropológica dental

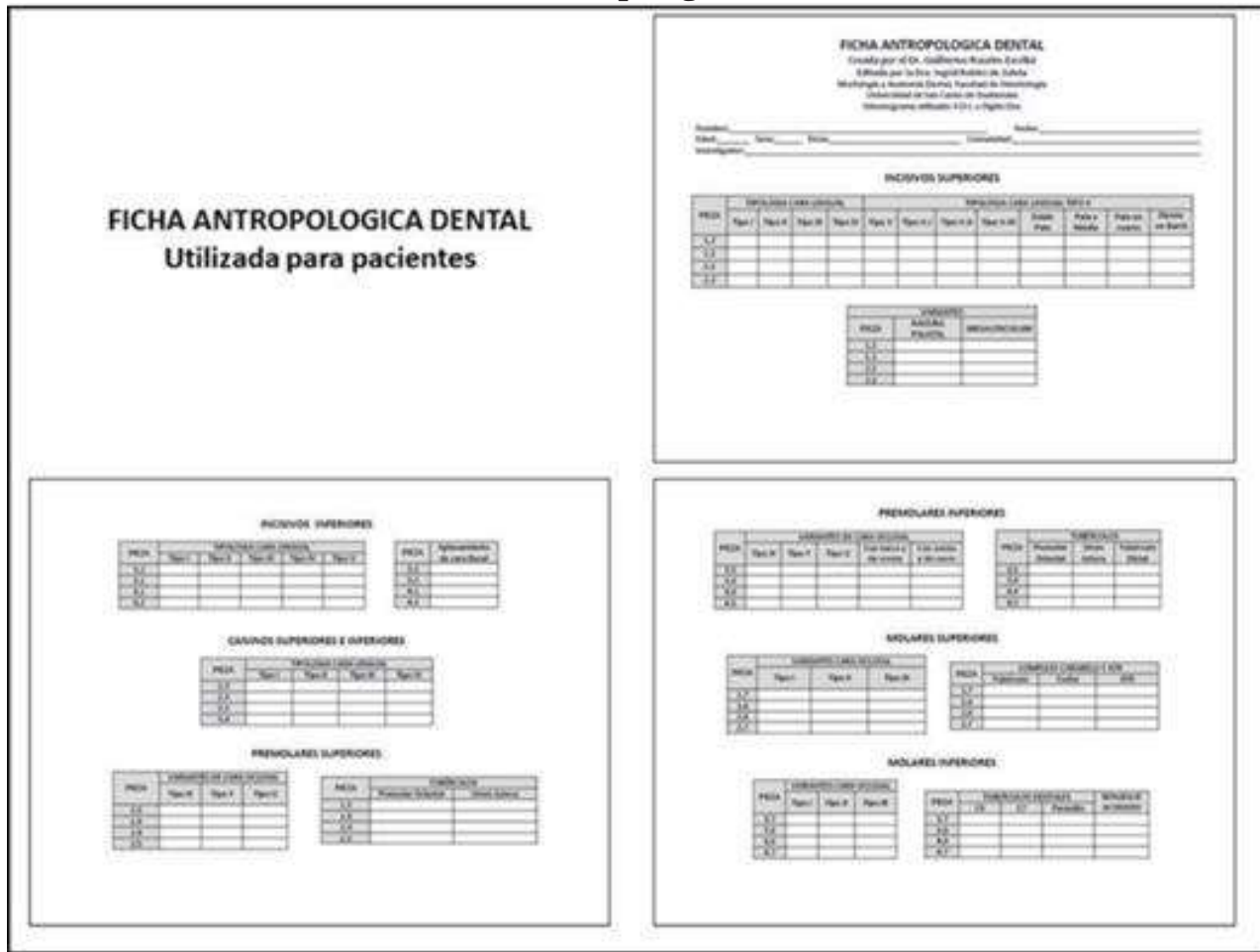

Fuente: Dr. Guillermo Rosales Escribá 
El formato original de estas fichas está hecho para utilizarse una por cada individuo, debido a que el presente estudio se hará con piezas sueltas, para la recolección de datos estas fichas fueron rediseñadas para poder ser utilizadas en la recolección de datos de piezas individuales y poder así agruparlas por grupo anatómico, no por individuo.

Las piezas dentales del maya prehispánico tanto anteriores como posteriores permanentes, fueron evaluadas y analizadas agrupándolas a través del sistema F.D.I. y colocando los datos obtenidos del análisis y evaluación en la ficha odontológica antes mencionada para determinar la frecuencia y prevalencia y en base a los datos obtenidos se procedió a sacar los promedios que reflejan la odontometría y morfología de las piezas dentales del maya prehispánico.

Para el presente estudio se tomaron en cuenta los siguientes criterios de inclusión y exclusión:

Criterios de inclusión:

- Piezas dentales permanentes anteriores completas apico-coronalmente

- Piezas dentales permanentes posteriores completas apico-coronalmente.

Criterios de exclusión:

- Restos radiculares o Fracturas coronales.

- Caries extensas o profundas que afecten un tercio o más de la corona dental.

- Terceros molares.

Piezas dentales deciduas.

- Enanismo radicular (excepto al evaluar variantes morfológicas. Fracturas radiculares (excepto al evaluar variantes morfológicas).

- Reabsorción radicular (excepto al evaluar variantes morfológicas

La muestra será determinada por la cantidad de piezas dentales proporcionadas por el Museo Nacional de Arqueología y Etnología de Guatemala (MUNAE), tomando como base los criterios de inclusión y exclusión ya establecidos.

\subsection{Equipo, instrumental y materiales utilizados}

Los materiales, instrumental y equipo utilizados durante la investigación son los siguientes:

- Piezas dentales permanentes del maya prehispánico proporcionadas por el MUNAE.

- Calibrador Digital Vernier 150MM/6" o Ficha

Odontométrica y ficha de Variantes Morfológicas.
Cámara Digital.

- Tela Dacrón Negra o Plasticina Negra.

Lápiz, borrador, lapiceros o Computadora.

Guantes.

Mascarillas.

Lentes protectores o Bata de laboratorio.

Documento de apoyo (Libro de texto de Anatomía y Morfología Dental).

\section{Calibrador Vernier}

Es un instrumento que sirve para medir las dimensiones y distancias tanto externas como internas.

Se trata de un aparato que permite tomar medidas más precisas que otros convencionales como las reglas. Además, posee un margen de error de tan solo $0.05 \mathrm{~mm}$ (0.0019 pulgadas)

La escala Vernier la invento Petrus Nonius, matemático portugués por lo que se le denomina “Nonius".

El diseño actual de escala deslizante debe su nombre al francés Pierre Vernier quien lo perfeccionó. Es un instrumento para medir longitudes que permite lecturas en milímetros y en fracciones de pulgada, a través de una escala llamada Nonio o Vernier. Está compuesto por una regla fija que es donde están graduadas las escalas de medición ya sea en milímetros, pulgadas o mixtas.

El calibrador vernier que será utilizado para la medición de las piezas del maya prehispánico, es de tipo digital de 150MM/6".

Las partes del calibrador son:

- Regla: Graduada en los sistemas métrico e inglés.

- Pata Fija: Con superficie de contacto a la pieza para medir exteriormente.

- Pata Móvil: Con superficie de contacto móvil a la pieza para medir exteriormente.

- Punta Fija: Parte fija de contacto con la pieza, para medir interiormente.

- Punta Móvil: Parte móvil de contacto con la pieza para medir interiormente.

- Impulsor: Apoyo del dedo pulgar para desplazar el cursor.

- Tornillo de Fijación o Freno: Fija la medida obtenida actuando sobre la lámina de ajuste.

- Nonio: Escala que otorga la precisión del instrumento según su cantidad de divisiones.

- Reglilla de Profundidad: Esta unida al cursor y sirve para tomar medidas de profundidad. 


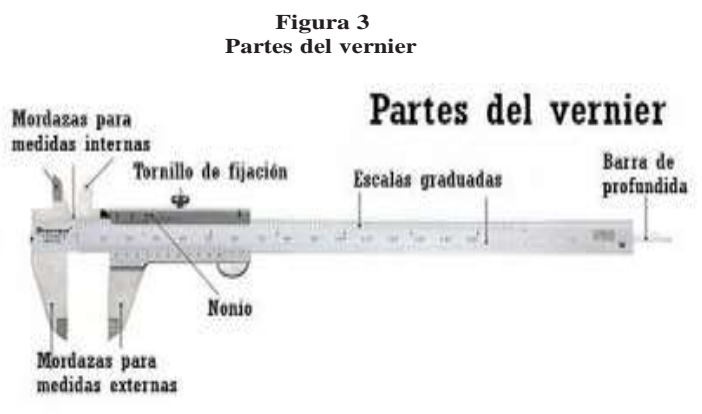

\section{MARCO TEÓRICO}

\subsection{LA CIVILIZACION MAYA}

Los mayas fueron una de las civilizaciones más grandes del mundo, se caracterizaron por su gran conocimiento intelectual; habitaron en lo que ahora es Honduras, Guatemala, Belice, El Salvador y Yucatán en México, desde el 2,000 a.C. hasta la colonización española 1,697 d.C. Eran personas de estatura mediana con desarrollo muscular, cara ancha, pómulos pronunciados, cabello negro y liso, frente ancha y nariz aguileña. La cultura maya se destacó a lo largo de 18 siglos en múltiples aspectos como su escritura jeroglífica, arte, arquitectura y sus notables sistemas de numeración, así como astronomía, matemáticas y comprensión de la ecología.

\section{a. El período formativo o preclásico (2000 a.c. a 300 d.c.)}

Esta etapa habría comenzado entre los años 2,000 y 1,500 a.C. y finalizado en el 300 d.C. A principios del Preclásico, las tierras altas estaban ocupadas por poblaciones protomayas y la costa pacífica por grupos proto-xincas, agrupados en pequeños pueblos de agricultores cercanos a fuentes de agua.

Con una afluencia olmeca marcada, entre 300 a.C. y $200 \mathrm{~d}$. C., se forman los primeros centros urbanos de Kaminaljuyú, AbajTakalik y el Baúl, en valles y próximos a tierras fértiles.

En este período se desarrollaron las primeras sociedades complejas y se cultivaron los alimentos básicos de la dieta maya: el maíz, el frijol, la calabaza y el chile, además se empiezan las construcciones de las primeras estructuras piramidales a lo largo de calzadas o alrededor de plazas, estas eran generalmente construcciones funerarias.

\section{b. El período clásico (300 a 1000 d.c.)}

Esta época comprende los siete siglos de mayor grandeza que tuvo esta civilización, se puede dividir en dos fases principales:

\section{c.El clásico Temprano (300 a 700 d.C.)}

Este período está caracterizado por la llegada de influencias culturales exteriores: Monte Albán, El Tajín y entre 400 y 500 d.C. una ola de influencia cultural teotihuacana. Penetrando la zona a través de las redes comerciales, los rasgos teotihuacanos se observan en la religión, la arquitectura y las artes pero también en los patrones de asentamiento y la organización socio-política. Se establece en las Tierras Altas una sociedad Teocrática caracterizada por la construcción de estructuras piramidales con tablero y talud, elevadas alrededor de piezas ceremoniales de tipo acrópolis.

Los mayas primero estuvieron ubicados en Petén, donde se encontraban las ciudades de Uaxactún y Tikal. Luego a finales del siglo IV los mayas iniciaron su expansión territorial, apareciendo nuevos núcleos urbanos, entre ellos Palenque, Piedras Negras, Copán, Uxmal, Bonampak, Sayil, Calakmul, el Mirador, Quiriguá y muchos otros.

En este periodo, aparecen también las primeras canchas de juego de pelota (abiertas o en palangana).

\section{d. El clásico Tardío (700 a 1000 d.C.)}

Las grandes ciudades persisten y pequeños centros cívicoceremoniales con edificios administrativos, pirámides, juegos de pelota, palacios y los primeros baños de vapor se multiplican en las pendientes de las lomas, próximas a las fuentes de agua.

El clásico tardío se caracteriza por una especialización regional en las producciones agrícolas y artesanales cuyos productos eran intercambiados en los mercados, regiones y a través de las amplias redes comerciales existentes tanto dentro de las Tierras Altas como con las Tierras Bajas del Petén en pleno apogeo.

La influencia teotihuacana disminuye tanto en la arquitectura como en las artes. Sin embargo, un nuevo flujo de grupos guerreros procedentes del Golfo de México penetra en la zona por el Norte.

Por otra parte, incursiones bélicas de grupos pipil-nicarao recién instalados en la franja pacífica, crean una situación de inseguridad. Al final del período, la situación política inestable se traduce por el abandono de los grandes centros cívicoceremoniales ubicados en los valles fértiles y la instalación de nuevos asentamientos en sectores defensivos, en las partes superiores de las colinas y las montañas. 
Estas ciudades alcanzaron su máximo esplendor hasta el siglo $X$, cuando comenzaron abandonarse inexplicablemente, moviéndose muchos de sus habitantes hacia el norte, a Yucatán, donde florecieron otras ciudades, como Chichen Itzá, Uxmal y Mayapán, grandes centros urbanos estos últimos, que también ya habían declinado cuando sus habitantes entraron en contacto con los conquistadores españoles, quienes posteriormente les dieron el nombre de Mayas.

\section{e. Historia de la odontología de los mayas}

La historia de la odontología maya se extendió por el sur de Yucatán, Guatemala y Honduras en el año 600 d.C. con una civilización que no consumía azúcar y que su sociedad tenía la costumbre de limpiarse los dientes después de las comidas. Según los rastros encontrados, para los mayas sus dientes y su sonrisa tenían una gran importancia y denotaba rango social y belleza.

Se cree que sus prácticas odontológicas tenían un carácter religioso y social, pero sobre todo estético; su tecnología era notable al observar las preparaciones dentales realizadas por ellos. Los mayas tenían la costumbre de aserrarse los dientes, dejándolos como una sierra, aparentemente simulando los dientes de un jaguar (figura 4), esta práctica se realizaba mediante ciertas piedras abrasivas, taladros rudimentarios empleando cuarzo como abrasivo; pero si por algún vestigio arqueológico es conocida esta civilización es por su capacidad de realizar incrustaciones o mutilaciones con piedras semipreciosas como jade, amatista, hematita, turquesa, cuarzo, cinabrio o pirita de hierro sin carácter médico lo cual denotaba galantería (figura 5).

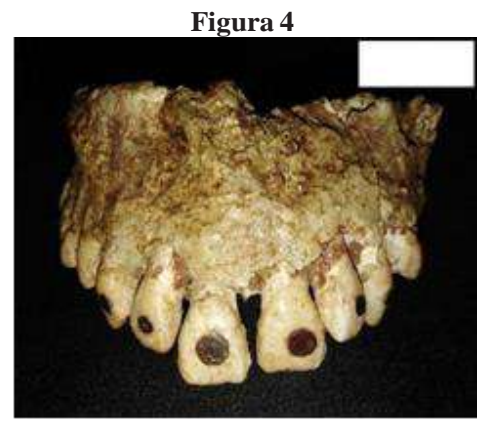

Fuente: Dr. Guillermo Rosales Escribá.

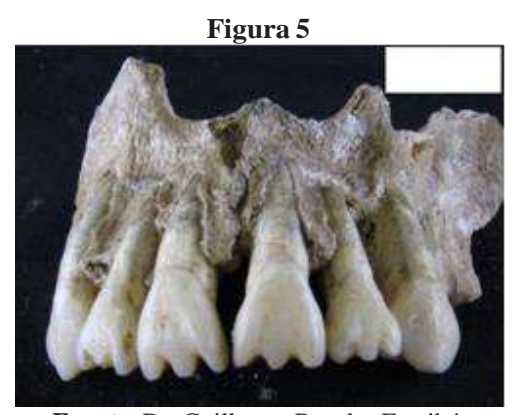

Fuente: Dr. Guillermo Rosales Escribá.
En las piezas examinadas en la presente investigación, propiedad del Museo de Arqueología y Etnología de Guatemala, (MUNAE), se pudieron observar múltiples piezas con este tipo de ornamentos, además de este tipo de práctica dental se hicieron notar desgastes o fracturas dentales ocasionadas por los hábitos de esta cultura, lo que hizo que muchas piezas no fueran evaluables en este estudio, estos desgastes eran debido básicamente a las costumbres de alimentación de esta civilización la cual consistía en una dieta a base de la ingesta del maíz y sus derivados (atoles, nixtamal, etc.), que al ser molido en utensilios hechos de piedra se incorporaban a los alimentos trozos de arena que sin querer generaban microfracturas y erosiones en las superficies dentales; aunque consumían productos locales como frijol, calabazas, raíces, frutas y productos de caza y pesca la dieta blanda en su mayoría rica en carbohidratos y pobre en vitamina $C$ provoco enfermedades periodontales dentro de la población maya; sin embargo era común masticar gomas, resinas y raíces de plantas para ayudar en la limpieza bucal.

A la llegada de los españoles hubo cambios en las costumbres de la población maya, entre las costumbres adquiridas estaba el consumir tabaco, al cual atribuían un uso limpiador, ya que no solamente lo fumaban sino lo masticaban, mezclándolo con conchas trituradas, generando así mayor cantidad de erosiones dentales, lo que hizo padecer de muchas otras enfermedades bucales.

Los mayas tenían un glifo para nombrar a las personas encargadas de realizar distintos trabajos odontológicos, un ejemplo de esto es la pieza de jade que se encuentra en el Museo del Jade en Costa Rica (figura 6), la cual se valúa su uso como un colgante posiblemente para el cuello que podría anunciar las cualidades de la persona que lo portase, esta imagen está compuesta por tres círculos que engloban al chamán-odontólogo que lo traducen como "El que produce sangría" y su paciente que es descrito como un buitre con vendaje anudado en la cabeza y es "El Glifo del dolor de Muela", entre ambos jeroglíficos y cerca de la cara del paciente está la representación parcial de una flor o de unos pétalos, que bien podrían ser de una planta medicinal para el tratamiento odontológico como sedante-analgésiconarcótico, se considera la posibilidad de que el glifo en esta imagen, sea la eventual representación de un antiquísimo proceso de atención dental, una imagen similar está presente en estelas ubicadas en la Gran Plaza de Copán Honduras (figura 7). 


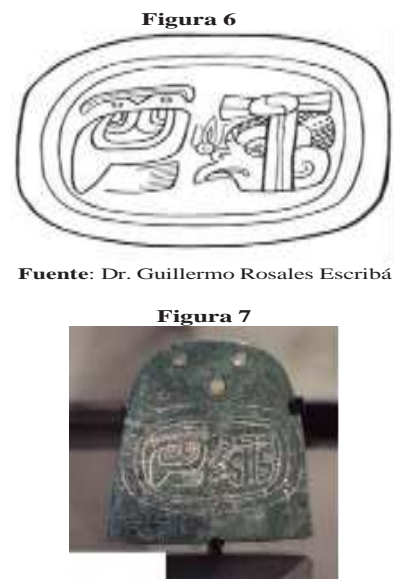

Fuente: Dr. Guillermo Rosales Escribá

\subsection{Odontometría}

Método de identificación que se basa en la obtención de medidas de uno o varios dientes que se hallan a disposición, además se aplica para el estudio de los parámetros morfométricos de los dientes. Es la determinación correcta de la longitud real del diente. Para este estudio de la dentición de mayas prehispánicos serán utilizadas cuatro medidas que son:

- Longitud Total o Largo Total del Diente (LT); ápicoincisal (AI) o ápico-oclusal. (AO).

1 Mesio-distal (MD).

- Buco-lingual (BL).

1 Cervico-incisal $(\mathrm{Cl})$ o Cervico Oclusal (CO).

Todos los dientes tienen tres dimensiones, al igual que todos los sólidos, los cuales son medibles estas dimensiones son:

1. Longitud o altura

- Anchura o latitud

1. Espesor.

Estas pueden subdividirse en distintos grados, que son máximo, parcial y mínimo.

\section{a. Longitud o altura}

Esta se divide en Máxima y Parcial.

\section{a.1 Longitud máxima:}

Esta es conocida como el largo total (LT) de la pieza dentaria. Los incisivos se miden desde el ápice (punta de la raíz) al borde incisal (punta del diente). Los dientes que tienen más de una raíz, o varias cúspides (en la corona), normalmente se específica el nombre de la raíz y la cúspide que se va a tomar como medida.
Para fines prácticos y que pueda ser repetitiva la medición deberán estar en contacto las cúspides con una de las partes activas del calibrador y se medirá la raíz más larga.

\section{a.2 Longitud parcial:}

Esta medida se divide en dos. La corona y la raíz.

Longitud de la Corona. Es la medida de la corona de la pieza dentaria, esta va del borde incisal/oclusal, al cuello del diente. Esta es una porción del diente cubierta de esmalte. Cuando se mide la corona, se realiza la medida cervico-incisal o cervico-oclusal. (Se utilizan las siglas $\mathrm{Cl}$ o $\mathrm{CO}$ ).

Longitud de la Raíz. Es la medida de la raíz de la pieza dentaria, esta va del ápice (punta de la raíz) al cuello del diente. Esta es una porción cubierta de cemento. Cuando se mide la raíz, se realiza la medida ápico cervical. (Se utilizan las siglas AC).
Figura 8
Piezas permanentes. Vista Frontal

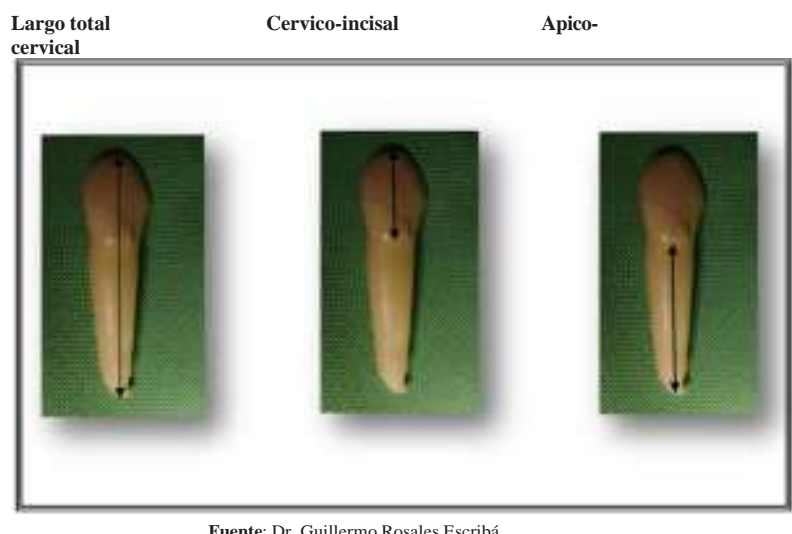

Fuente: Dr. Guillermo Rosales Escribá

Figura 9

Molares superior e inferior con dimensiones Cérvico-Oclusal y Largo Total

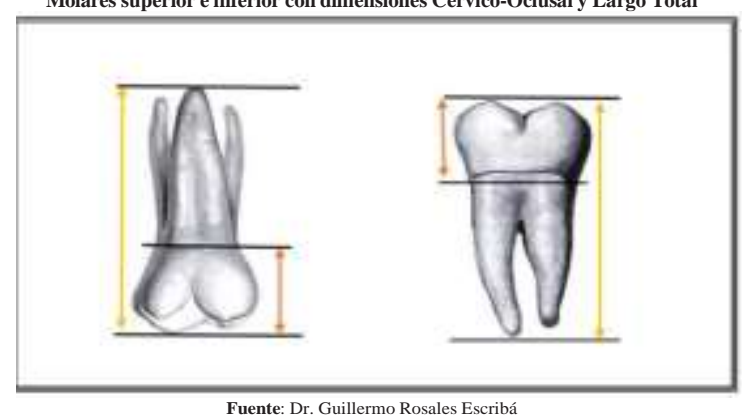

Anchura o latitud.

Esta palabra es sinónimo de medir el diámetro mesio-distal de las piezas dentarias (la simbología utilizada es MD), esta es la medida del ancho de la corona del diente, va de un lado al otro lado del diente. Se conoce como la medida de mesial a distal del diente. En el diámetro mesio-distal puede realizarse tres mediciones: 
Ancho máximo de la corona. Es la parte más convexa de los lados del diente. (Esta medida sería realmente el diámetro mesiodistal total del diente).

Borde cortante. Esta se clasifica como una medida parcial (anchura parcial), esta es la parte cortante del diente, que es el borde incisal en piezas incisivas o la oclusal en piezas premolares y molares.

Ancho Mínimo. Puede decirse que es el ancho en el cuello del diente (Cérvix).

Figura 10

Piezas permanentes. Vista Frontal. Diámetro Mesio-Distal Canino Inferior Molar Inferio

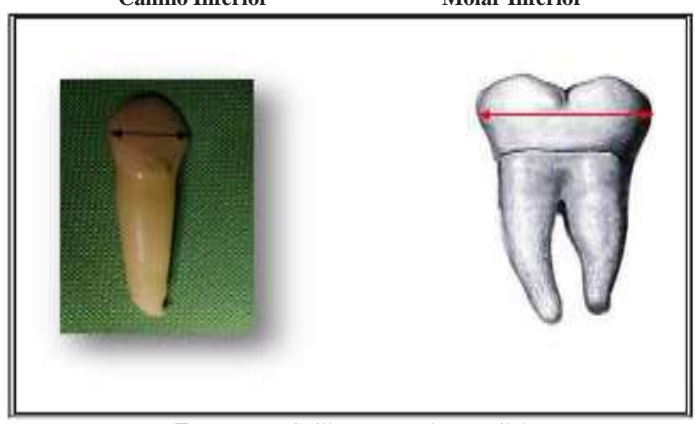

Fuente: Dr. Guillermo Rosales Escribá

Espesor. Esta es la medida buco lingual de la pieza, algunos autores le llaman también vestíbulo lingual (se abrevia BL, que significa buco-lingual). Esta es la medida del grosor del diente, va de bucal a lingual (de adelante hacia atrás).

Esta se divide en tres espesores: espesor mínimo o espesor máximo y espesor parcial (cuello).

\section{Figura 15}

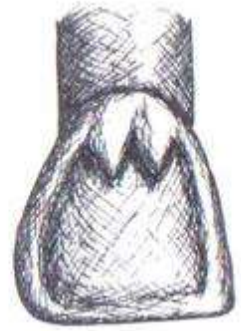

Fuente: Dr. Guillermo Rosales Escribá

Espesor mínimo. Esta medida se realiza en el borde cortante de la pieza dentaria.

Espesor Máximo. Esta es la medida de la parte más ancha en grosor del diente.

Espesor Parcial. Esta se realiza a nivel del cuello del diente. Es conocida como una dimensión parcial, conocida como la parte de transición entre el esmalte y el cemento.
Figura 12

Distribución de piezas dentales permanentes Maxilar superior - maxilar inferior

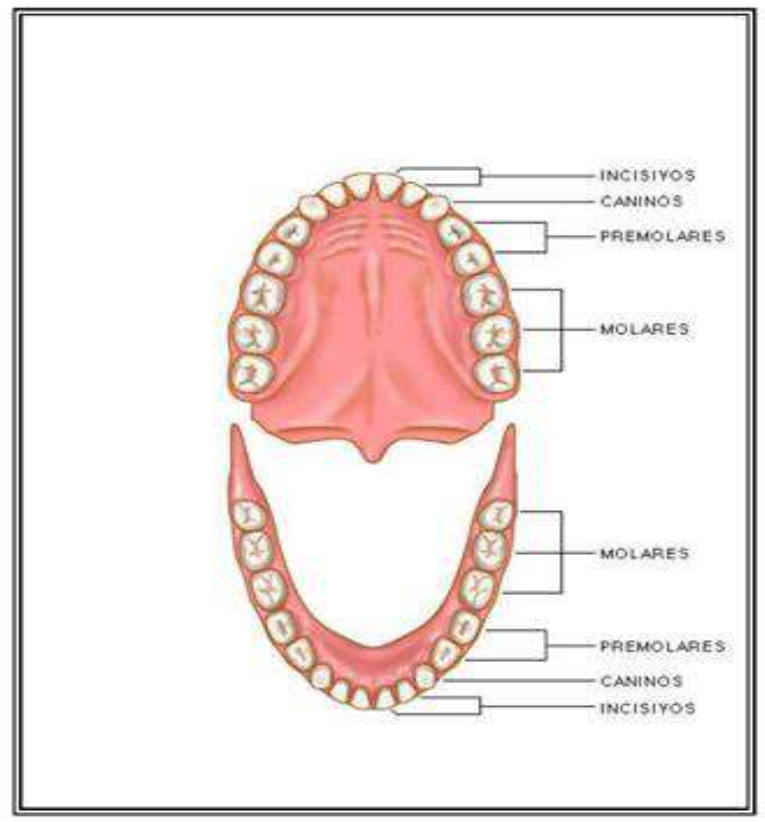

Fuente: Dr. Guillermo Rosales Escribá

8 incisivos, 4 caninos, 8 premolares, 12 molares.

\subsection{Variantes morfológicas}

En muchas ocasiones observamos en los dientes ciertas características que no son comunes a todas las piezas dentarias, ni a todas las personas; sin embargo, en el mayor número de los casos las pasamos desapercibidas sin preguntarnos, ¿qué es aquella depresión o elevación?; ¿si existe en solo esa pieza o si puede darse como una característica general de un grupo de ellas?

Además, en muchas oportunidades las consideramos como anomalías y no como lo que realmente son variantes morfológicas.

Cabe entonces definir lo que es una variante: "Es una diferencia de mayor o menor grado que no alteran esencialmente la forma del diente, pero sí sus caracteres generales lo suficiente para individualizarlo", a diferencia de lo que es una anomalía: "Variación que ocurre rara vez y que puede alterar fundamentalmente la forma del diente.

- Las variantes morfológicas dentales generalmente se presentan de forma bilateral.

- Las variantes morfológicas dentales no muestran una predilección marcada por sexo.

- Debe entenderse que existen variantes morfológicas que manifiestan una frecuencia 
más alta hacia determinado grupo étnico en relación a otro.

- No se ha encontrado en los distintos estudios realizados, alguna variante morfológica que sea más frecuente de encontrar en dientes preliminares que en dientes permanentes.

- Las variantes morfológicas dentales deben considerarse como un factor de apoyo coadyuvante, pero no como una forma definitiva y única en la identificación de grupos étnicos específicos.

5.4 Variantes de cara lingual de incisivos superiores e inferiores

Estas variantes fueron descritas por primera vez por G. V. Black, y están dadas por: la presencia o ausencia de la proyección del cíngulum hacia incisal, prominencia de los rebordes marginales y por la profundidad de la fosa lingual.

A diferencia de los grupos dentarios posteriores, premolares y molares, las variantes de cara lingual de las piezas anteriores se observan generalmente iguales, en incisivos centrales y laterales de la misma arcada.

Algunos autores como Woelfel le llaman a la proyección del cíngulum rebordes linguales accesorios.

\section{Tipo I}

Es aquella cara lingual que presenta poca concavidad, tiene poco prominentes sus rebordes marginales, sin que exista ningún límite entre éstos y el cíngulum. El cíngulum no posee ninguna proyección hacia incisal. Es el tipo más común en incisivos centrales y laterales inferiores, no importando el origen étnico de la población.

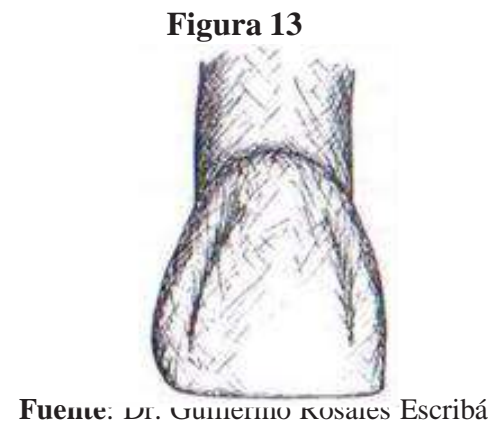

Tipo II

Es la cara lingual en la que la fosa lingual se encuentra dividida por la proyección hacia incisal del cíngulum; la cual en este tipo es UNICA, de forma triangular de base cervical y vértice incisal. La fosa lingual es más profunda que la que posee el tipo I y sus rebordes más pronunciados. En esta pieza la parte más cóncava de la fosa lingual se encuentra a nivel del tercio cervical, siendo el tercio incisal de la misma su parte plana.

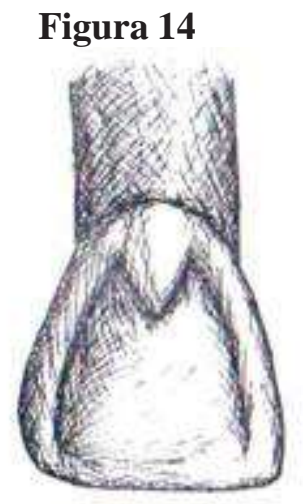

Fuente: Dr. Guillermo Rosales Escribá

Tipo III

Es la cara lingual que presenta características similares a las del tipo II con la diferencia que el cíngulum presenta dos proyecciones hacia incisal, razón por la que el tercio cervical de la fosa se encuentra dividido en tres porciones. Generalmente las prolongaciones del cíngulum (rebordes linguales accesorios) son más cortas que las que presenta el tipo II.

Figura 15

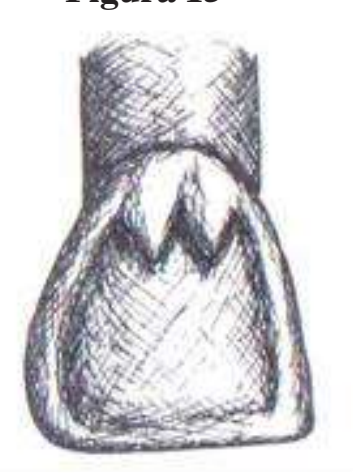

Fuente: Dr. Guillermo Rosales Escribá

Tipo IV

Es similar a los tipos II y III excepto que hay tres prolongaciones de cíngulum hacia incisal, de las cuales la central es la más larga. Este tipo presenta una superficie muy rugosa. Es el tipo menos frecuente de todos los tipos. 
Figura 16

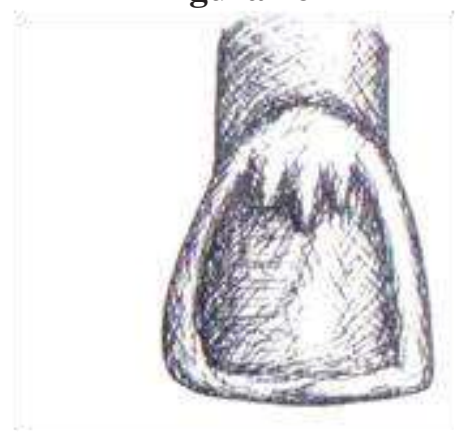

Fuente: Dr. Guillermo Rosales Escribá

Tipo V

Difiere de los anteriores, en que sus rebordes marginales son extremadamente pronunciados, creciendo tanto en ancho como en grosor conforme más se acercan al tercio cervical.

La prominencia de los rebordes hace que la fosa lingual se observe muy profunda. En la unión de los dos rebordes a nivel del tercio cervical se encuentra un pozuelo que recibe el nombre de

Agujero Ciego o Foramen Coecum.

Además presenta el borde incisal más grueso y ancho que los otros tipos. Es considerado el diente más prevalente de razas orientales, razón por lo que es poco frecuente en negros y caucásicos, siendo más común encontrarlo en amerindios.

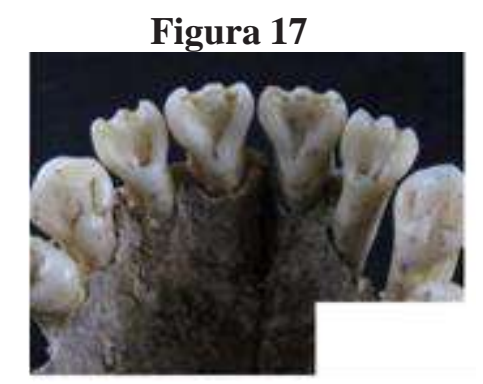

Fuente: Dr. Guillermo Rosales Escribá

En algunas oportunidades el tipo $\mathrm{V}$ puede presentar una o dos prolongaciones hacia incisal, a estas dos variantes el $\mathrm{Dr}$. Guillermo Rosales las ha clasificado en Tipo V-I y Tipo V-II.

Tipo V-I

Es aquella variante de cara lingual de incisivos en la cual la pieza muestra una forma clásica de diente en pala, pero presenta una prolongación del cíngulum hacia incisal.
Figura 18
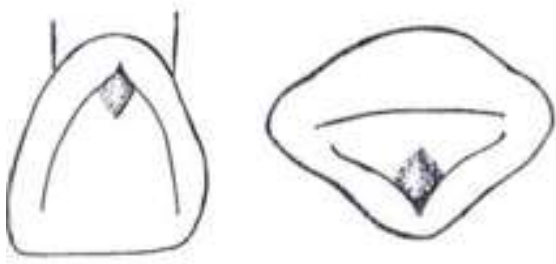

Fuente: Dr. Guillermo Rosales Escribá

Tipo V-II

Es aquella variante de cara lingual de incisivos en la cual la pieza presenta una forma clásica de diente en pala, pero se observan dos prolongaciones del cíngulum hacia incisal.

\section{Figura 19}
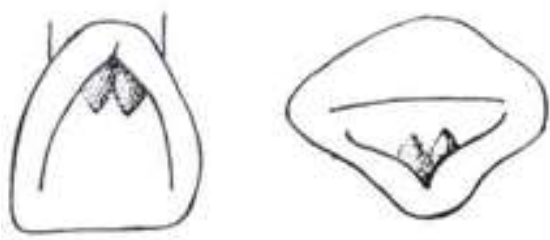

Fuente: Dr. Guillermo Rosales Escribá

Ranura linguoproximal

Es una depresión lineal que se localiza en la cara lingual, tercio cervical, dividiendo el o los rebordes marginales del cíngulum de los incisivos centrales y laterales superiores. La ranura puede presentarse tanto sobre el reborde proximal mesial o distal, o sobre ambos.

Es más frecuente encontrarla en los incisivos laterales superiores que en los demás incisivos y más aún en aquellos incisivos en los que se presenta el tipo $\mathrm{V}$ de cara lingual. En ocasiones la ranura es tan larga que se continúa con la raíz, ocasionando en estoscasos, problemas periodontales a las piezas donde se encuentra.

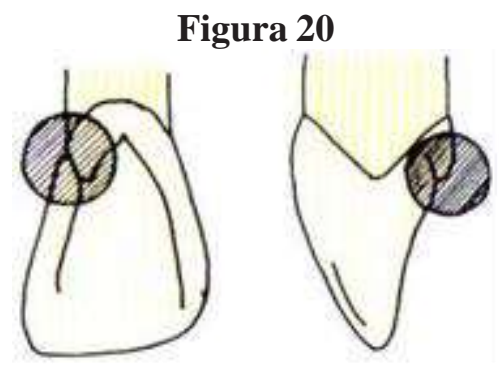

Fuente: Dr. Guillermo Rosales Escribá 
Megacingulo.

Es la variante que se caracteriza por un excesivo crecimiento de la prolongación del cíngulum hacia lingual y/o incisal. Esta variante ha sido nombrada por autores como Scott y Turner II como Tubérculo Dental, Tubérculo Dentale o Tuberculum Dentale.

Esta variante es más frecuente de aparecer en incisivos superiores tanto central como lateral. Siendo el incisivo lateral superior permanente la pieza más frecuente en que se ha encontrado. Además en el megacíngulum se va a encontrar, casi siempre, una prolongación de la pulpa, por lo que puede ocasionar problemas pulpares. Esta variante no se ha encontrado en dientes preliminares.

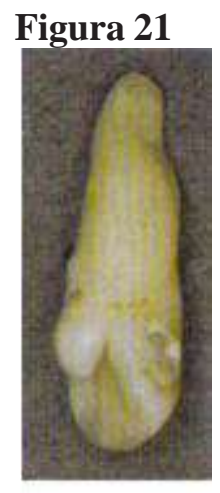

Fuente: Dr. Guillermo Rosales Escribá

\section{Diente en barril}

Es aquella variante que se presenta en incisivos superiores permanentes tanto en centrales como en laterales. Es más frecuente en el incisivo lateral superior y de forma unilateral. No se presenta en incisivos inferiores ni en dientes preliminares.

Se caracteriza por el aumento de volumen de rebordes proximales hacia lingual, dándole una forma cilíndrica a la pieza.

\section{Figura 22}

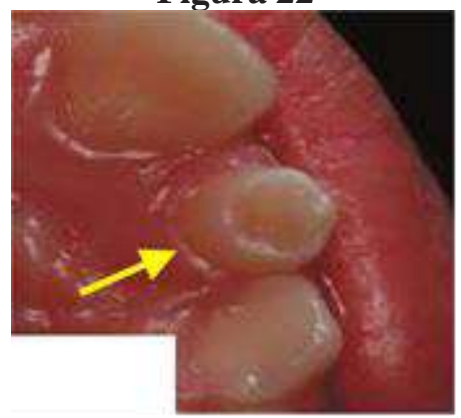

Fuente: Dr. Guillermo Rosales Escribá
5.5Variantes de cara lingual de caninos superiores e inferiores Clasificacion Dr. Guillermo Rosales Escribá

Las variantes van a depender de la presencia o ausencia de: reborde mediano lingual, fosa o fosetas linguales y del tubérculo triangular de Mühlreiter.

Tipo I

Es aquella variante que presenta un cíngulum desarrollado pero el lóbulo mediano no lo es tanto, por lo consiguiente su fosa lingual es poco profunda, limitada por los rebordes proximales, de constitución robusta.

Figura 23

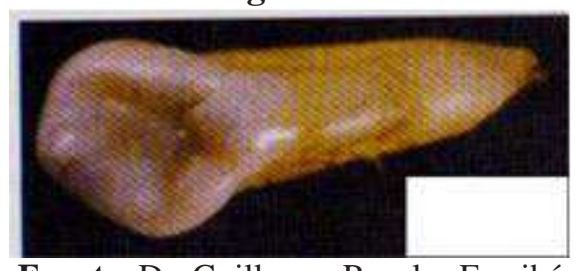

Fuente: Dr. Guillermo Rosales Escribá

Tipo II

El cíngulum y el lóbulo mediano se desarrollan a tal grado que se une en el tercio medio de la cara y dan como resultado la formación de un reborde mediano lingual.

Esta estructura divide a la fosa lingual en dos fosetas: una mesiolingual y la otra distolingual.

Figura 24

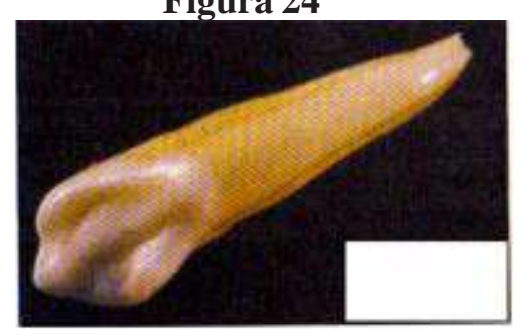

Fuente: Dr. Guillermo Rosales Escribá

Tipo III

La descripción de este tipo es idéntica a la del tipo II, pero en la foseta distolingual aparece un pequeño tubérculo de forma triangular cuya base se encuentra en el brazo distal del borde incisal.

Este tubérculo es llamado: tubérculo triangular, tubérculo triangular de Mühlreiter o tubérculo de Mühlreiter.

Figura 25

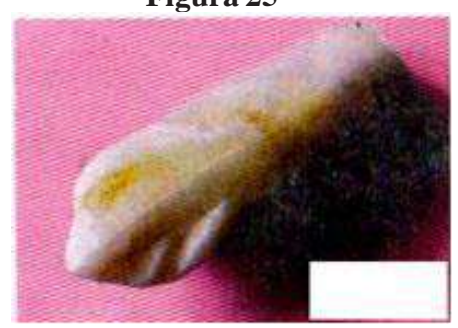

Fuente: Dr. Guillermo Rosales Escribá 
Tipo IV

Es aquel que presenta una marcada concavidad marginada por amplios rebordes marginales y un pozuelo en lugar de la prolongación del cíngulum. Presenta unos rebordes proximales muy desarrollados y fosa lingual profunda.

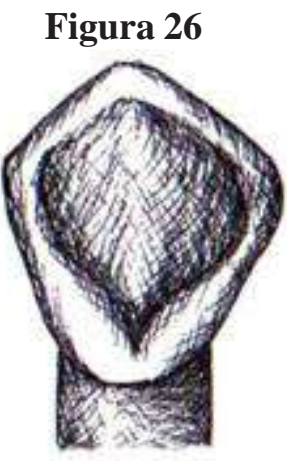

Fuente: Dr. Guillermo Rosales Escribá

5.6 Variantes de caras oclusales de premolares

Las variantes morfológicas de los premolares superiores e inferiores, están dadas por la configuración de los surcos en la cara oclusal y por el número de sus cúspides linguales.

Tipo $\mathrm{H}$

Tienen dos cúspides: una bucal y una lingual, siendo la cúspide bucal más alta.

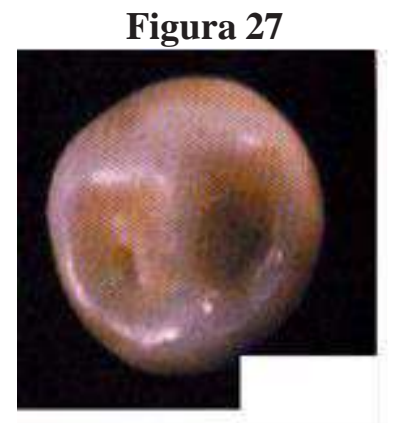

Fuente: Dr. Guillermo Rosales Escribá

Tipo U

Es aquel premolar que se caracteriza por poseer pequeñas cúspides por lingual, separadas entre sí por ranuras primarias. Su surco principal tiene forma de $U$, siendo la cúspide bucal más alta y prominente que las cúspides linguales.

\section{Figura 28}

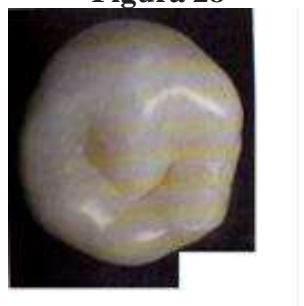

Fuente: Dr. Guillermo Rosales Escribá
Tipo $Y$

Es el premolar que presenta tres cúspides: una bucal y dos linguales

Figura 29

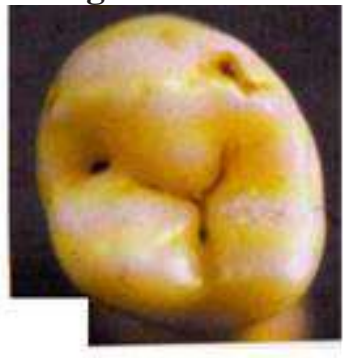

Fuente: Dr. Guillermo Rosales Escribá

El Dr. Guillermo Rosales ha clasificado a los premolares de otra manera:

Tipo I:

Con surco y sin cresta.

\section{Figura 30}

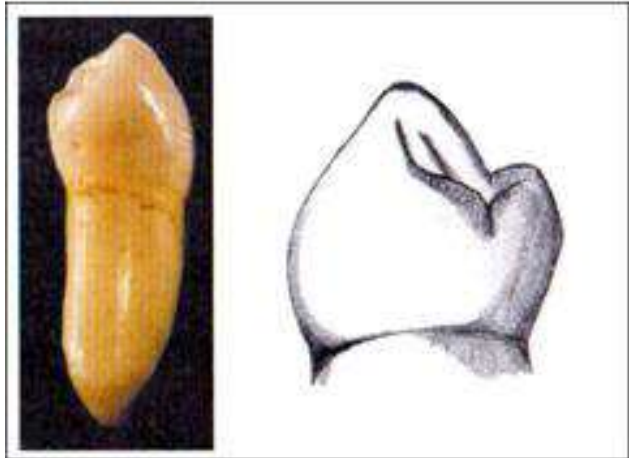

Fuente: Dr. Guillermo Rosales Escribá

Tipo II:

Sin cresta y con surco

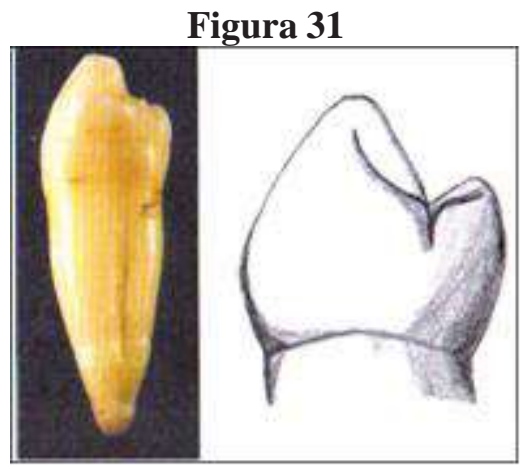

Fuente: Dr. Guillermo Rosales Escribá

Odontoma evaginado

Es una elevación tubercular que se encuentra en la superficie oclusal de los premolares, ocupando casi siempre el lugar de la fosa central en premolares inferiores y sobre el reborde triangular bucal en premolares superiores y sobrepasando la altura cuspídea. 
Figura 32

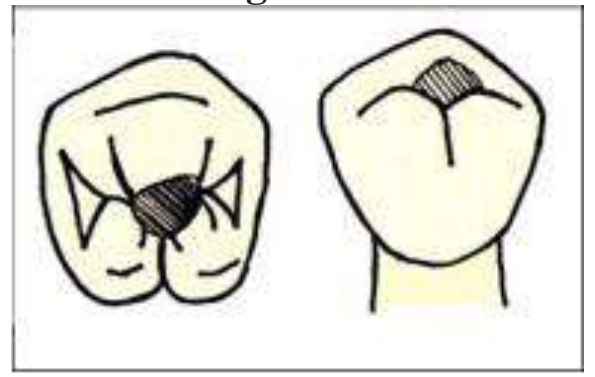

Fuente: Dr. Guillermo Rosales Escribá

\section{Odontoma evaginado}

Es una elevación tubercular que se encuentra en la superficie oclusal de los premolares, ocupando casi siempre el lugar de la fosa central en premolares inferiores y sobre el reborde triangular bucal en premolares superiores y sobrepasando la altura cuspídea.

\section{Figura 33}

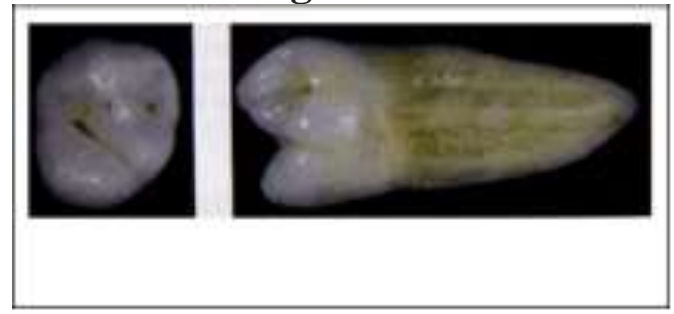

Fuente: Dr. Guillermo Rosales Escribá

\section{Utom azteca}

Se caracteriza por el adosamiento de una de una especie de diente paramolar a la superficie proximal, tanto corona como raíz de los premolares

\section{Figura 34}

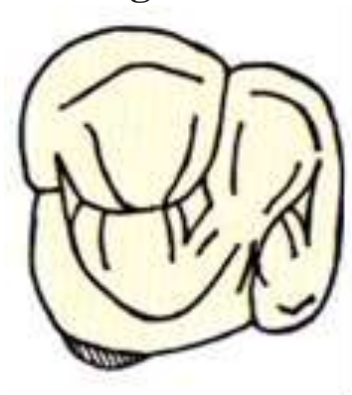

Fuente: Dr. Guillermo Rosales Escribá

\section{Figura 35}

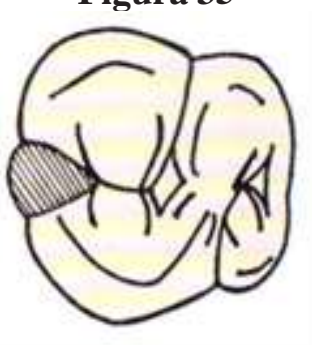

Fuente: Dr. Guillermo Rosales Escribá

Reborde transversal anterior o ATR

Se trata de una pequeña elevación lineal más o menos desarrollada de forma triangular, que se encuentra en los molares superiores, en la que su vértice coincide con el vértice de la foseta triangular mesial y la base sobre el reborde marginal correspondiente. Es más frecuente encontrarlo en el primer molar superior permanente, que en el segundo o tercero.

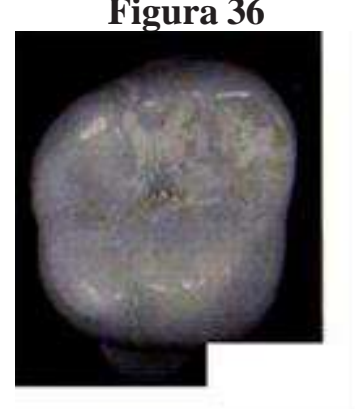

Fuente: Dr. Guillermo Rosales Escribá

5.7 Variantes de molar superior

La disminución del tamaño mesiodistal de la cúspide distopalatina determina la aparición de distintos tipos de cara oclusal.

Forma romboidal o tipo I

Recuerda la forma del primer molar, aunque la cuarta cúspide se halla un poco disminuida. Las cuatro cúspides están perfectamente diferenciadas, al igual que los rebordes marginales.

\section{Figura 37}

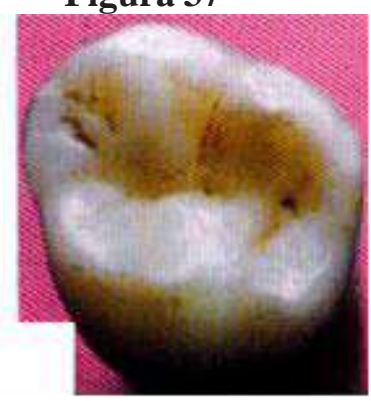

Fuente: Dr. Guillermo Rosales Escribá 


\section{Figura 38}

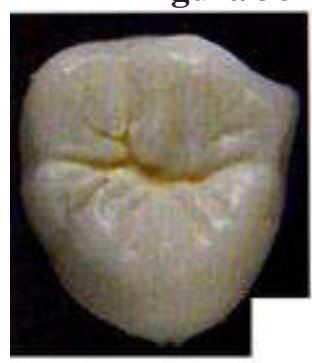

Fuente: Dr. Guillermo Rosales Escribá

Forma trapezoidal o tipo II

El diámetro mesiodistal disminuye en la parte palatina por la reducción de la cuarta cúspide. A veces desaparece el surco secundario que la separa del reborde marginal y ambos quedan fusionados.

\section{Figura 39}

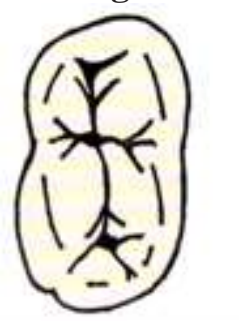

Fuente: Dr. Guillermo Rosales Escribá

5.8 Variantes morfológicas de molares inferiores

Tipo I

El surco mesiobucal se origina en relación mesial al surco lingual. Por esta razón la cúspide mesiobucal es más angosta en sentido mesiodistal que la cúspide mesiolingual y la cúspide distobucal queda centrada en relación a las otras cúspides.

\section{Figura 40}

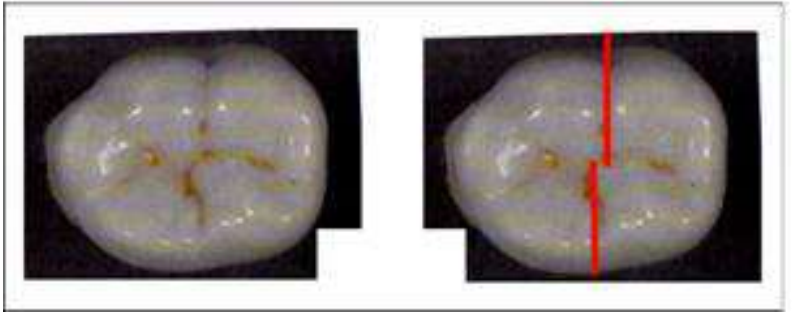

Fuente: Dr. Guillermo Rosales Escribá

Tipo II

El surco mesiobucal y el surco lingual coinciden por lo que la cúspide mesiobucal y la mesiolingual queda en el mismo ancho mesiodistal. Es la forma más común en la que se presentan los molares inferiores de cuatro cúspides.
Figura 41

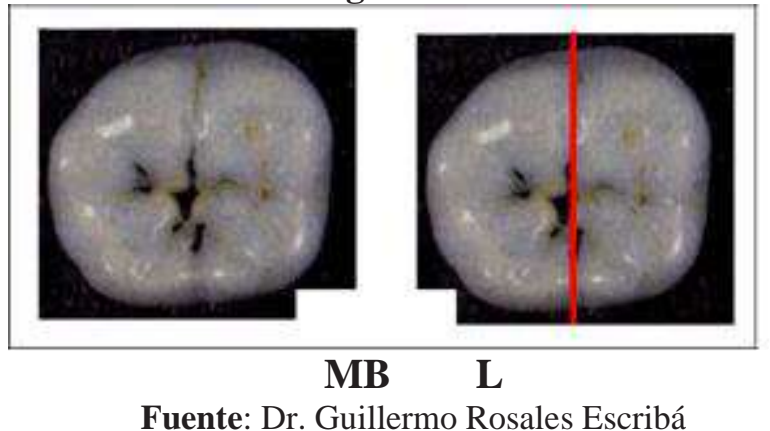

Tipo III

Se presenta en las piezas en las que el surco mesiobucal se encuentra distal al lingual, por esta razón la cúspide mesiobucal resulta mucho más grande en ancho que todas las demás cúspides.

Figura 42

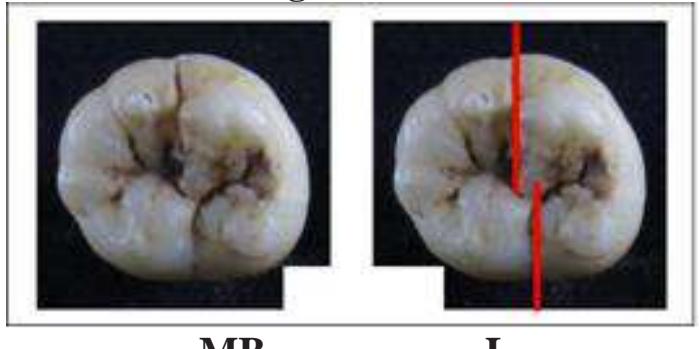

MB

L

Fuente: Dr. Guillermo Rosales Escribá

Tuberculum sextum $\mathrm{O} \mathrm{C} 6$

Es una elevación poco pronunciada situada en un lugar equidistante de las dos cúspides distales de los molares inferiores pentacuspídeos.

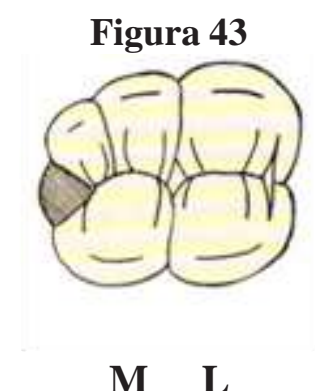

Fuente: Dr. Guillermo Rosales Escribá

Tuberculo de selenka O C7

Es una elevación pequeña que se localiza entre las dos cúspides linguales de los molares inferiores.

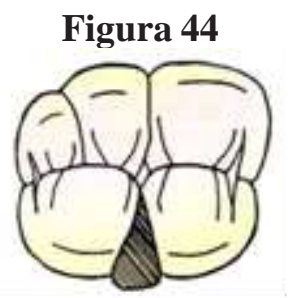

Fuente: Dr. Guillermo Rosales Escribá 

Paramolares de bolk

Es una elevación que se localiza en la cara bucal tercio mesial de los segundos y terceros molares superiores.

Puede encontrarse en tres formas distintas:

\section{a. Tubérculo Paramolar}

Cuando se encuentra adherido a la corona de las piezas y presenta solo esmalte en su composición.

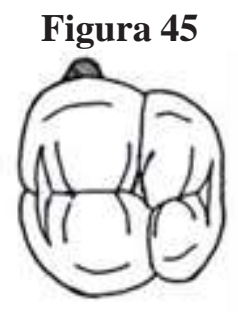

Fuente: Dr. Guillermo Rosales Escribá

b. Diente paramolar adherido

Cuando se encuentra adherido a la corona de los dientes pero encontrándose esmalte y cemento en su composición.

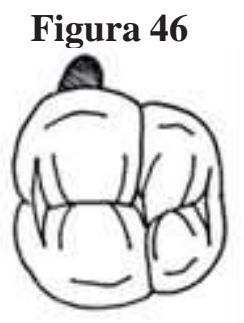

Fuente: Dr. Guillermo Rosales Escribá

\section{c. Diente Paramolar Libre}

Cuando se encuentra totalmente separado de la pieza dentaria. Ubicándose en la parte bucal de los molares.

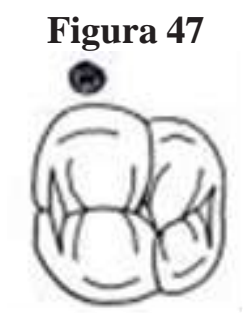

Fuente: Dr. Guillermo Rosales Escribá

\section{Parastilo}

Se caracteriza por la presencia de un tubérculo sobre la superficie bucal, generalmente de la cúspide mesiobucal de molares inferiores permanentes. También puede presentarse en molares inferiores preliminares.

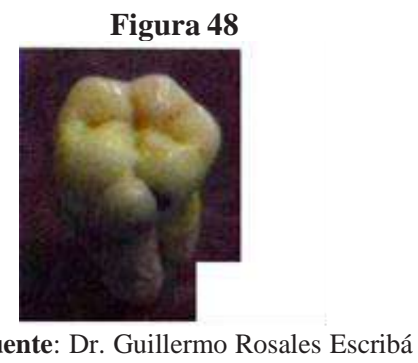

Radix

Es una extensión de las raíces de los molares inferiores, ya sea hacia bucal o hacia lingual. Generalmente se presenta solo en una raíz de la pieza, ya sea la mesial o la distal, siendo la raíz distal la que lo presenta con más frecuencia.

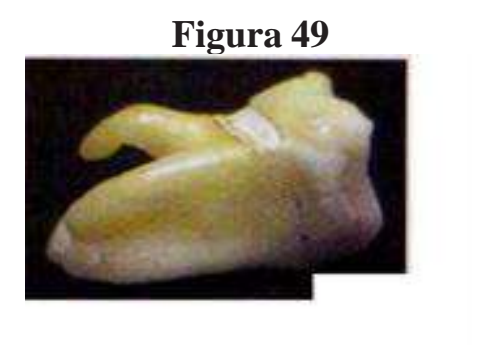

Fuente: Dr. Guillermo Rosales Escribá

\section{RESULTADOS}

Para poder determinar la odontometría y las variantes morfológicas de las piezas dentales de los mayas prehispánicos se realizó un trabajo de campo en las instalaciones del Museo Nacional de Arqueología y Etnología de Guatemala, MUNAE, dicha faena se llevó a cabo en tres sesiones en las cuáles se realizaron mediciones en todas las piezas que cumplieran con los criterios de inclusión y exclusión descritos anteriormente y se evaluaron las características morfológicas de cada pieza tanto sueltas como en cráneos, siendo esto motivo por el cual la muestra utilizada para el examen morfológico fue mayor.

El Arqueólogo Josué García, fue el designado por parte del MUNAE para proporcionarnos los bienes arqueológicos que fueron seleccionados para realizar el presente estudio el cuál contenía las piezas dentales sueltas y los cráneos propiedad del museo, los mismos provenían de diferentes sitios arqueológicos y abarcan distintos puntos del territorio nacional, proporcionando mayor información y sustento a la presente investigación. La muestra fue proveniente de:

- Poptún, Petén. 
Uaxactún, Petén.

El Remate Flores, Petén.

Nebaj, Quiche.

San Agustín Acasaguastlán, El Progreso.

Kaminaljuyú/San Jorge, Guatemala.

Tiquisate, Escuintla.

Entierros sin hcer mención de su ubicación geográfica.

- Lugares Desconocidos.

Resultados de odontometría en piezas permanentes del maya prehispánico del MUNAE 2,019

A continuación se presentan los resultados de los cuadros estadísticos que contienen la odontometría de las piezas dentales permanentes del maya prehispánico realizadas durante el trabajo de campo en el MUNAE, en este estudio fueron medidas, estudiadas, analizadas y aceptadas bajo los criterios de inclusión y exclusión una muestra universo de 273 piezas dentales proveniente de los diferentes sitios arqueológicos. Se llevaron a cabo las mediciones del largo total de las piezas dentales (inciso/oclusal-apical, mesiodistal, buco-lingual e inciso/oclusal-cervical), además a cada muestra de las piezas dentales de acuerdo a su grupo anatómico se les realizó un análisis estadístico que permitiera definir las características que en general poseen las piezas dentales del maya prehispánico.

\section{Odontometría de incisivos centrales superiores}

Se observan las medidas promedio de los incisivos centrales superiores encontrados en los mayas prehispánicos, tomando como base para determinar la confiabilidad de la muestra, la desviación estándar del largo total de las piezas nos arroja un \pm 1.76 lo que nos da una confianza cercana al $95 \%$, presenta también la media del largo total de las piezas de $23.40 \mathrm{~mm}$, la media mesio-distal de $8.80 \mathrm{~mm}$, la media buco-lingual de $7.32 \mathrm{~mm}$ y la media cervico-incisal de $10.26 \mathrm{~mm}$, tomada de una muestra de 28 piezas dentales.

\section{Odontometría de incisivos laterales superiores}

Se presentan los promedios de los incisivos laterales superiores del maya prehispánico con una media del largo total de $22.24 \mathrm{~mm}$ y una desviación estándar de \pm 1.81 lo que representa una confianza cercana al 95\%, la media mesio-distal de $7.38 \mathrm{~mm}$, la media buco-lingual de $6.56 \mathrm{~mm}$ y la media cervico-incisal de $9.52 \mathrm{~mm}$, en una muestra de 22 piezas dentales.
Odontometría de caninos superiores

Se presenta la mayor cantidad de piezas evaluadas en el estudio de odontometría siendo la muestra de 45 piezas dentales y el promedio de caninos superiores del maya prehispánico con una media del largo total de $27.03 \mathrm{~mm}$ y una desviación estándar de \pm 2.03 , lo que nos da una confianza cercana al 95\%, la media mesio-distal de $8.34 \mathrm{~mm}$, la media buco-lingual de $8.81 \mathrm{~mm}$ y la media cervico-incisal de $10.73 \mathrm{~mm}$. Siendo estas piezas las de mayor tamaño del largo total en el presente estudio.

\section{Odontometría de primeros premolares superiores}

Se observan las medidas promedio de los primeros premolares superiores encontradas en los mayas prehispánicos, tomando como base para determinar la confiabilidad de la muestra estudiada, la desviación estándar del largo total de las piezas nos arroja un \pm 1.85 lo que nos da una confianza cercana al 95\%, los datos obtenidos de la media mesio-distal es de $7.80 \mathrm{~mm}$, la media buco-lingual de $9.77 \mathrm{~mm}$ y la media cervicoincisal de $8.17 \mathrm{~mm}$, tomada de una muestra de 20 piezas dentales.

\section{Odontometría de segundos premolares superiores}

Se presenta la menor cantidad de piezas superiores evaluadas en el estudio de odontometría siendo la muestra de 7 piezas dentales y el promedio de los segundos premolares superiores del maya prehispánico con una media del largo total de $21.29 \mathrm{~mm}$ y una desviación estándar de \pm 1.59 , lo que nos da una confianza cercana al $95 \%$, la media mesio-distal de $7.42 \mathrm{~mm}$, la media buco-lingual de $9.70 \mathrm{~mm}$ y la media cervico-incisal de $7.66 \mathrm{~mm}$.

\section{Odontometría de primeros molares superiores}

Se observan las medidas promedio de los primeros molares superiores del maya prehispánico, siendo la media de la longitud total de estas piezas evaluadas de $20.13 \mathrm{~mm}$ con una desviación estándar de \pm 1.49 lo que nos da una confianza cercana al 95\%, la media mesio-distal de $11.01 \mathrm{~mm}$, la media buco-lingual de 12.07 y la media cervico-incisal de $7.70 \mathrm{~mm}$, tomada de una muestra de 29 piezas dentales.

\section{Odontometría de segundos molares superiores}

La media del largo total de los segundos molares superiores del maya prehispánico es de $20.09 \mathrm{~mm}$, con una desviación estándar de \pm 1.95 lo que representa una confianza cercana al $95 \%$, la media mesio-distal de $9.50 \mathrm{~mm}$, la media buco-lingual 
de $11.49 \mathrm{~mm}$ y la media cervico-incisal de $7.42 \mathrm{~mm}$, tomada de una muestra de 16 piezas dentales.

\section{Odontometría de incisivos centrales inferiores}

Se observan las medidas promedio de los incisivos centrales inferiores encontrados en los mayas prehispánicos, tomando como base para determinar la confiabilidad de la muestra, la desviación estándar del largo total de las piezas nos arroja un \pm 1.78 lo que nos da una confianza cercana al $95 \%$, la media mesio-distal de $6.08 \mathrm{~mm}$, la media buco-lingual de $6.20 \mathrm{~mm}$ y la media cervico-incisal de $9.07 \mathrm{~mm}$, tomada de una muestra de 15 piezas dentales.

\section{Odontometría de incisivos laterales inferiores}

Se presenta la menor cantidad de piezas inferiores evaluadas en el estudio de odontometría siendo la muestra de 7 piezas y el promedio de los incisivos laterales inferiores del maya prehispánico con una media del largo total de $21.29 \mathrm{~mm}$ siendo de igual tamaño que el largo total los segundos premolares superiores y una desviación estándar de \pm 3.03 , lo que nos da una confianza cercana al $99 \%$, la media mesiodistal de $6.22 \mathrm{~mm}$, la media buco-lingual de $6.37 \mathrm{~mm}$ y la media cervico-incisal de $9.58 \mathrm{~mm}$.

\section{Odontometría de caninos inferiores}

Se observan las medidas promedio de los caninos inferiores encontrados en los mayas prehispánicos, tomando como base para determinar la confiabilidad de la muestra, la desviación estándar del largo total de las piezas nos arroja un \pm 2.15 lo que nos da una confianza cercana al 95\%, la media mesio-distal de $7.66 \mathrm{~mm}$, la media buco-lingual de $8.15 \mathrm{~mm}$ y la media cervico-incisal de $11 \mathrm{~mm}$, tomada de una muestra de 11 piezas dentales.

\section{Odontometría de primeros premolares inferiores}

Se observan las medidas promedio de los primeros premolares inferiores encontrados en los mayas prehispánicos, tomando como base para determinar la confiabilidad de la muestra, la desviación estándar del largo total de las piezas nos arroja un \pm 1.62 lo que nos da una confianza cercana al $95 \%$, la media mesio-distal de $7.55 \mathrm{~mm}$, la media buco-lingual de $8.53 \mathrm{~mm}$ y la media cervico-incisal de $7.98 \mathrm{~mm}$, tomada de una muestra de 29 piezas dentales.

\section{Odontometría de segundos premolares inferiores}

Se observan las medidas promedio de los segundos premolares inferiores encontrados en los mayas prehispánicos, tomando como base para determinar la confiabilidad de la muestra, la desviación estándar del largo total de las piezas nos arroja un \pm 1.46 lo que nos da una confianza cercana al $95 \%$, la media mesio-distal de $7.80 \mathrm{~mm}$, la media buco-lingual de

$8.54 \mathrm{~mm}$, siendo esta medida muy similar a la media de los primeros premolares inferiores buco-lingual de $8.53 \mathrm{~mm}$, y la media cervico-incisal de $7.57 \mathrm{~mm}$, tomada en una muestra de 15 piezas dentales.

\section{Odontometría de primeros molares inferiores}

Se observan las medidas promedio de los primeros molares inferiores del maya prehispánico, siendo la media de la longitud total de estas piezas evaluadas de $20.94 \mathrm{~mm}$ con una desviación estándar de \pm 1.70 que nos da una confianza cercana al $95 \%$, la media mesio-distal de $12.04 \mathrm{~mm}$, la media buco-lingual de $11.02 \mathrm{~mm}$ y la media cervico-incisal de $7.58 \mathrm{~mm}$, tomada de una muestra de 19 piezas dentales.

\section{Odontometría de segundos molares inferiores}

Se observan las medidas promedio de los segundos molares inferiores del maya prehispánico, siendo la media de la longitud total de estas piezas evaluadas de $19.95 \mathrm{~mm}$ con una desviación estándar de \pm 1.64 que nos da una confianza cercana al $95 \%$, la media mesio-distal de $11.45 \mathrm{~mm}$, la media buco-lingual de $10.77 \mathrm{~mm}$ y la media cervico-incisal de $7.17 \mathrm{~mm}$, tomada de una muestra de 10 piezas dentales.

Luego de recopilar los datos odontométricos de las piezas evaluadas en este estudio se obtuvieron los datos que componen la siguiente tabla que será útil para las conclusiones de esta investigación.

Tabla No. 1

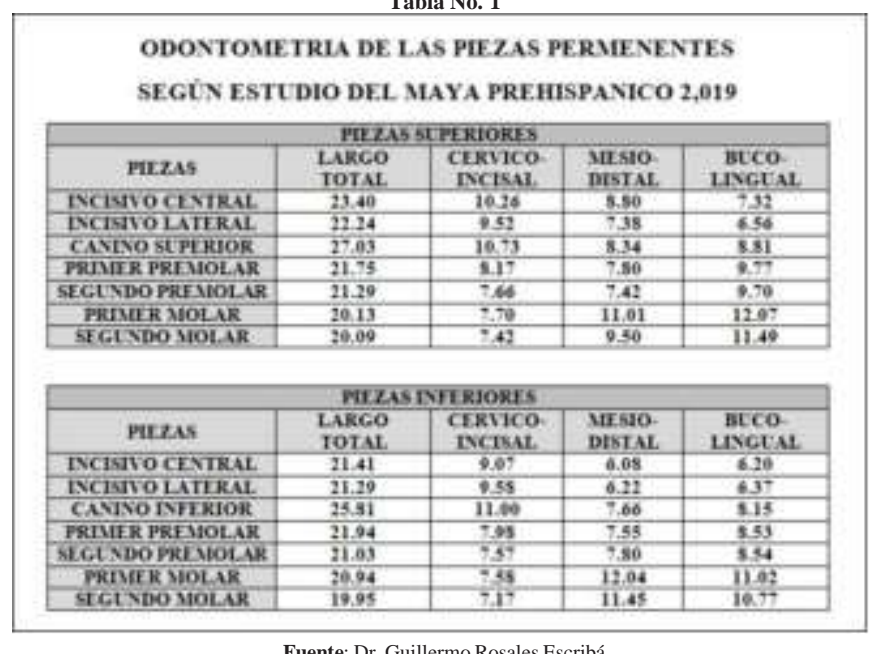

Fuente: Dr. Guillermo Rosales Escribá 
Resultados de variantes morfológicas de las piezas dentales permanentes de mayas prehispánicos del MUNAE 2,019

Las piezas usadas para la evaluación de variantes morfológicas en el Museo Nacional de Arqueología y Etnología de Guatemala fueron un total de 529 piezas dentales, se utilizaron piezas sueltas y piezas en cráneos que cumplieran los requisitos de inclusión.

A continuación se describen los resultados de este trabajo fundados en los cuadros de los resultados que se encuentran en anexos, estos fueron tomados de las de las fichas antropológicas dentales que fueron utilizadas en la presente investigación.

Variantes morfológicas de incisivos centrales superiores

Podemos notar que el $80.56 \%$ de los incisivos centrales superiores, es decir 4/5 de la muestra es de tipo V; un $13.89 \%$ fue tipo V-II y un 5.55\% fue pala y media. Solamente a un $2 \%$ de estas piezas evaluadas se le encontró ranura palatal.

Variantes morfológicas de incisivos laterales superiores

Al igual que en el incisivo central superior, en este cuadro

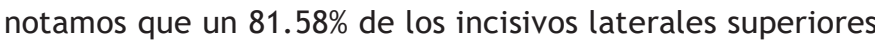
fueron tipo V, el tipo V-I con un $15.79 \%$ y un $2.63 \%$ fue en barril. En este grupo se encontró que el $5.4 \%$ de la muestra presentó ranura palatal.

Variantes morfológicas de caninos superiores

El 56\% de los caninos superiores evaluados fueron de tipo I; el $26 \%$ tipo II y el $18 \%$ de tipo III.

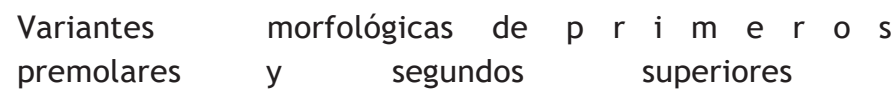

El total de premolares estudiados, no importando si fueran primeros o segundos el $100 \%$ de las piezas fue del tipo $\mathrm{H}$.

Variantes morfológicas de primeros molares superiores

Del total de primeros molares superiores estudiados, el $90 \%$ presentó el tipo I y el $10 \%$ de las piezas presentó el Tipo II. Con respecto al Complejo Carabelli, únicamente 2 piezas, equivalente al $4 \%$ presentaron la fosita y un $12 \%$ se encontró ATR, no encontrándose ninguno de los otros elementos: tubérculo, ranura linguoproximal o ranura oclusomesial.

Variantes morfológicas de segundos molares superiores En segundos molares superiores se presentaron los tres tipos
I, II y III. El tipo II fue el más frecuente con un $47.61 \%$ y el tipo III fue el segundo en frecuencia con un $38.1 \%$, el menos frecuente de los tres tipos fue el tipo I con un $14.29 \%$.

\section{Variantes morfológicas de incisivos centrales inferiores}

Del total de incisivos inferiores estudiados 15 piezas, el $100 \%$ presentó el tipo I

Variantes morfológicas de incisivos laterales inferiores

En los incisivos inferiores se encontraron dos variantes, el tipo I y V. Siendo el tipo I el más frecuente con un $93.33 \%$ del total de piezas estudiadas.

Variantes morfológicas de caninos inferiores

En los caninos inferiores, se encontró que el tipo más frecuente fue el tipo II. Sin embargo también se presentaron el tipo I con un $16.15 \%$ y el tipo III con un $12.9 \%$

Variantes morfológicas de primeros premolares inferiores

En los primeros premolares inferiores la variante más frecuente fue Con Cresta y sin surco con un porcentaje del $84 \%$, la variante con Surco y sin cresta se presentó en un $10 \%$, el tipo $Y$ se presentó en un $2 \%$ y el tipo $U$ se encontró en un $4 \%$. El tubérculo distal estuvo presente en el $14 \%$ de los primeros premolares inferiores examinados.

Variantes morfológicas de segundos premolares inferiores

En los segundos premolares inferiores la variante más frecuente fue el tipo $\mathrm{H}$ con un $48.48 \%$, la segunda variante en frecuencia fue el tipo $U$ siendo el tipo $Y$ el tercero en frecuencia, además se presentó el tipo Con Cresta y sin surco, siendo esta variante más característica de primeros que de segundos premolares, esta variante es la menos frecuente de todas en los segundos premolares inferiores.

Variantes morfológicas de primeros molares inferiores

En los primeros molares inferiores, la variante de cara oclusal más frecuente fue el tipo II con un $72.58 \%$, el tipo I fue el segundo en frecuencia, y el menos frecuente de todos fue el tipo III con un 3.23\%. De los tubérculos el Sextum obtuvo una frecuencia de $14.51 \%$ y el tubérculo de Selenka se encontró en un $3.23 \%$ de la muestra examinada.

Variantes morfológicas de segundos molares inferiores

En los segundos molares inferiores, la variante de cara oclusal 
más frecuente fue el tipo II con un $86.48 \%$, e I tipo I y el tipo III se encontraron casi con la misma frecuencia siendo el tipo III, ligeramente más alto con un $8.11 \%$, y el tipo I el menos frecuente con $5.41 \%$.

De los tubérculos presentes en los molares, solo se encontró el Sextum con un $10.82 \%$.

\section{CONCLUSIONES}

Luego de la realización de la presente investigación se llegó a varias conclusiones tanto en odontometría como en variantes morfológicas propias de cada grupo anatómico de piezas dentales los cuales se detallan a continuación:

Conclusiones de odontometria de las piezas dentales permanentes del maya prehispánico 2,019

La pieza dental que presentó la mayor medida en el largo total del estudio del maya prehispánico fue el canino superior con $27.03 \mathrm{~mm}$.

La pieza dental que presentó la menor medida en el largo total del estudio del maya prehispánico fue el segundo molar inferior con $19.95 \mathrm{~mm}$.

La pieza dental que presentó la mayor medida mesio-distal del estudio del maya prehispánico fue el primer molar superior con $11.01 \mathrm{~mm}$.

La pieza dental que presentó la menor medida mesio-distal del estudio del maya prehispánico fue el incisivo central inferior con $6.08 \mathrm{~mm}$.

La pieza dental que presentó la mayor medida buco-lingual del estudio del maya prehispánico fue el primer molar superior con $12.07 \mathrm{~mm}$.

La pieza dental que presentó la menor medida buco-lingual del estudio del maya prehispánico fue el incisivo central inferior con $6.20 \mathrm{~mm}$.

La pieza dental que presentó la mayor medida cervico-incisal del estudio del maya prehispánico fue el canino inferior con $11 \mathrm{~mm}$.

La pieza dental que presentó la menor medida buco-lingual del estudio del maya prehispánico fue el segundo molar inferior con $7.17 \mathrm{~mm}$.

La medida promedio que representa la media del largo total de las piezas dentales del estudio del maya prehispánico coinciden en tamaño el segundo premolar superior y el incisivo lateral inferior con $21.29 \mathrm{~mm}$, siendo ambas muestras de 7 piezas dentales, existiendo diferencia en la desviación estándar del segundo premolar superior de \pm 1.59 lo que indica una confianza cercana al $68 \%$ y el incisivo lateral inferior una desviación estándar de \pm 3.03 lo que indica una confianza cercana al $99 \%$, lo que demuestra que las medidas tomadas de esta pieza dental fueron más uniformes que las antes mencionadas.

La medida promedio que representa la media mesio-distal de las piezas dentales del estudio del maya prehispánico coinciden en tamaño el primer premolar superior y el segundo premolar inferior con $7.80 \mathrm{~mm}$, la muestra del primer premolar superior fue 20 piezas dentales y la muestra del segundo premolar inferior fue 15 piezas dentales, la desviación estándar del primer premolar superior es de \pm 0.37 y el segundo premolar inferior es de $\pm 1.13 \%$ lo que indica que los resultados del segundo premolar inferior fueron más confiables ya que es un indicativo que tuvo menor variación en sus dimensiones.

Debido al origen de este estudio la muestra fue de tipo universal ya que las piezas por su procedencia eran limitadas, en algunos grupos dentales se tuvo una muestra poco significativa como lo fue el incisivo lateral inferior y el segundo premolar superior que tuvieron una muestra de 7 piezas cada uno.

Durante el presente estudio se tomaron en cuenta las tablas de odontometría de G.V. Black (año 1,890), así como también las de Julian B. Woelfel (año 1,997), para poder determinar si se presentaba alguna variación odontométrica significativa con el presente estudio de odontometría en el maya prehispánico. A lo que se concluyó que en la tabla que corresponde a las piezas permanentes del estudio del maya prehispánico comparadas con las tablas antes mencionadas, no presentan alguna diferencia significativa en tamaño, mostrando un leve aumento en las dimensiones de la mayoría de las piezas dentales en el estudio del maya prehispánico.

Conclusiones de sobre las variantes morfológicas de las piezas dentales permanentes del maya prehispanico 2,019

Los incisivos superiores tanto centrales como laterales presentaron únicamente el tipo V. El tipo V-I y V-II se encontró en un $13.89 \%$.

El diente en barril se encontró en un $2.63 \%$ y únicamente en incisivos laterales superiores.

En incisivos superiores la ranura palatal se encontró en un 
alrededor del 5\% tanto en centrales como laterales.

En caninos superiores la variante más frecuente de cara lingual fue el tipo III.

En caninos superiores la variante más frecuente de cara lingual fue el tipo I con un $56 \%$, la segunda variante en frecuencia fue el tipo II con un $26 \%$ y la menos frecuente fue el tipo III con un $18 \%$, no se encontró ninguna pieza que tuviera la variante tipo IV o canino en pala.

En los premolares superiores tanto primeros como segundos premolares solo presentaron el tipo $\mathrm{H}$ en un $100 \%$ en ambas piezas.

En los primeros molares superiores la variante de cara oclusal más frecuente fue el tipo I con un $90 \%$ y el tipo II se encontró en un $10 \%$.

En los segundos molares superiores la variante de cara oclusal más frecuente fue el tipo II con un $47.61 \%$, sin embargo el tipo III presentó una frecuencia del $38.1 \%$, siendo el tipo I de cara oclusal la variante menos frecuente de todas con un $14.29 \%$.

Con respecto al Complejo de Carabelli solamente se encontró la fosita en 2 primeros molares superiores equivalente al 4\%, no se encontraron ninguno de los otros componentes del Complejo de Carabelli, ni tampoco se encontró el Complejo en segundos molares superiores.

El Reborde Transversal Anterior o ATR solo se encontró en primeros molares superiores con una frecuencia del $12 \%$.

En incisivos centrales inferiores la única variante de cara lingual presente en todas las piezas fue el tipo I.

En laterales inferiores se encontraron dos variantes de cara lingual, siendo el tipo I el más frecuente con un $93.33 \%$ y el tipo $\mathrm{V}$ con un $6.67 \%$.

En caninos inferiores la variantes de cara lingual más frecuente fue la tipo II con un 70.97\%, y tanto el tipo I con un 16.13 y el tipo III con un $12.9 \%$ fueron las variantes que ocuparon el segundo y tercer lugar en frecuencia. Al igual que en el canino superior, no se encontró ninguna pieza que presentara el tipo IV o canino en pala.

En los primeros premolares inferiores la variante de cara oclusal más frecuente fue Con Cresta y Sin Surco con un porcentaje del $84 \%$.

El tubérculo distal se encontró en un $14 \%$ de los primeros premolares inferiores estudiados, no encontrándose en segundos premolares inferiores.

En segundos premolares inferiores se encontraron los tres tipos de cara oclusal, siendo el tipo $\mathrm{H}$ el más frecuente con un $48.48 \%$ seguido del tipo $U$ con un $30.3 \%$ y el tipo $Y$ fue la variante morfológica menos frecuente de las tres con un $12.12 \%$. Un dato interesante de hacer mención es que se encontraron tres segundos premolares inferiores que presentaron la variante Con Cresta y Sin surco, esta variante es más frecuente de encontrarse en primeros premolares inferiores.

La variante de cara oclusal más frecuente que se encontró en primer molar inferior fue la tipo II con un $72.58 \%$ de los casos estudiados, el tipo I se encontró en un $24.19 \%$ y se encontraron tres piezas equivalente al $3.23 \%$ que presentaron el tipo III.

En segundos molares inferiores el tipo más frecuente fue el tipo II con un $86.48 \%$, el tipo III se presentó en 3 piezas, equivalente al $8.11 \%$ y el menos frecuente fue el tipo II con un $5.41 \%$.

Con respecto a los tubérculos de Selenka y Sextum, en primeros molares inferiores el tubérculo Sexto se en encontró en 9 piezas, equivalente al 14.51\%. En segundos molares inferiores el C6 se presentó en 4 piezas lo que representó un $10.82 \%$ El tubérculo de Selenka únicamente se encontró en primeros molares inferiores con una frecuencia del $3.23 \%$.

\section{Recomendaciones}

Luego de concluido este trabajo se recomienda realizar un estudio basado en el guatemalteco actual, ya que hasta el día de hoy no se cuenta con datos propios de la región, lo cual será de utilidad para determinar de manera más exacta las dimensiones y variantes dentales a nivel regional y corroborar de alguna manera este estudio.

\section{Agradecimientos}

Agradecemos de manera especial por su colaboración en la presente investigación al Dr. Guillermo Rosales, a las autoridades del MUNAE en especial al Arqueólogo Josué García, al Ing. Diego Fernando Valle y a la Dra. Ingrid Robles de Zuleta por su valiosa colaboración en la elaboración de este trabajo.

\section{REFERENCIAS}

\begin{tabular}{|c|c|c|c|c|}
\hline Barahona, & C. & U., & es, & J. \\
\hline $\begin{array}{l}\text { González, } \\
\text { Oliveira. }\end{array}$ & $\begin{array}{l}\text { E., } \\
\text { O. }\end{array}$ & Moncada, & $\begin{array}{l}\text { G., } \\
\text { Mé-todos }\end{array}$ & $\&$ \\
\hline
\end{tabular}


De Los Individuos. Re-visión De Tema. Revista Facultad De Odontología Universidad De Antioquia, 29(1), 1

- 14. Obtenido de http://www.scielo.org.co/pdf/rfoua/v2 9n1/0121-246X-rfoua-29-01-00173.pdf

Correa Ramírez, A. I. (1990). Identificación Forense. México: Trillas. Demirjian, A., Golds-tein, H., \& Tanner, J. (mayo de 1973). A New System Of Dental Age Assessment. Human Biology(45:2), 211 - 227.

Escobar, M. (1992). Odontología Pediárica (2da ed.). (E.Universitaria, Ed.) Santiago, Chile. Iannucci, J. M., \& Jansen Howerton, L. (2016). Dental Radiography Principles And Tecniques (5th. ed.). Columbus, Ohio, E.E.U.U: Elsevier.

INACIF. (24 de mayo de 2017). Identificación Humana Forense. Obtenido de inacif.gob.gt: http://www.inacif. gob.gt/index. php/therapies/k2-blog/item/31-laidentificacion-humana-forense

Instituto Nacional de Estadistica. (diciembre de 2014). Instituto Nacional De Estadística De Guatemala (INE). (INE,Ed.) Obtenido de https://www.ine.gob.gt/index.php /est adisticas: https://www.ine.gob.gt/sistema/u p loas / $2015 / 07 / 20 /$ y YXFscGDOuzX zAzSVWOzGnaa1WSaqajj.pdf

Instituto Nacional De Medicina Legal y Ciencias Forenses. (2011).17 Reglamento Técnico Para La Estimación de Edad En Clínica Forense. Bogotá, Colombia. Obtenido de

http://www.medicinalegal.gov.co/ documents/20143/40696/Reglam ento + T\% C $3 \% A 9$ cnico + para + la + est $i$ $m$ a ci $\%$ C $3 \% B 3 n+d e+e d a d+e n+C l \% C$ $3 \%$ A D n i c a + Forense +Versi $\%$ C $3 \%$ B 3 $\mathrm{n}+3+\mathrm{D}$ ic i e m bre +de+2011.pdf/ 6 b f 6 1c74-3789-e215-f1ab-445b2681ad07

Krenzer, U. (2006). Compendio De Métodos Antropológico Forenses (1era. ed., Vol. IV (Es-timación de la edadosteológica en subadultos)). Guatemala, Guatemala. Obtenido de http://.cafcaguatemala.org

Liversidge, H. (10 de agosto de 2012). The Assessment And Interpretation Of Demirjian, Goldstein And Tanner's Dental Maturity. Annals Of Human Bioogy, 20.doi: $10.3109 / 03014460.2012 .71$ 6080

Mincer, H., Harris, E., \& Barryman , H. (marzo de 1993). The A.B.F.O.Study Of Third Molar
Development And Its Use An Estimator Of Chronological Age. Journal Of Forensic Sciences, 38(2), $379-390$.

Moorrees, C., Fanning, E., \& Hunt, E. (noviembre de 1963). Age Variation Of Formation Stages For Tem Permanent Teeth. Journal of Dental Research(42), 1940 - 1502. Nolla, C. (1960). The Development Of The Permanent Teeth. Journal Of Dentistry For Children, 27, 254 - 266.

Robledo Acinas, M., \& Shánchez Sháncehez, J. (Abril - Mayo de 2013). Estimación de la edad en niños. Estudio radiológico de tobillo. Laboratorio de Antropología Forense y Criminalística (7). Obtenido de https: / / www.uv.es/gicf/4A2_Robledo_GICF_07.pdf

Rodríguez Cuenca, J. (2004). La Antropología Forense en la Identificación Humana (1era. ed.). Bogotá, Colombia: Editora Guadalupe Ltda. Rosales Escribá, $\quad$ G . (2015). Morfología y Anatomía Dental (7ma. ed.). Guatemala, Guatemala, Guatemala: LR Publicidad \& Diseño.

Rosales Escribá, G. (2015). Morfología Y Anatomía Dental (7ma.ed.). Guatemala, Guatema-la: LR Publicidad \& Diseño. Santos Hernández, J. R. (2010). Enciclopedia Criminalística, Criminología e Investigación (1era. Edición ed., Vol. II). Bogotá, Colombia: Sigma Editores.

Schour, l., \& Massler, M. (1941). The Development Of The Human Dentition. JADA(42), 1153-1160. Smith, H. (1991). Standards Of Human Tooth Formation And Dental Age Assessment. En K. Larsen (Ed.), Advances in Dental Anthropology (págs. 143-168). Michigan, E.E.U.U.

Whaites, E., \& Drage, N. (2013). Dental Radiography And Radiology (5ta.ed.). Londres, Inglaterra: Elsevie Ldt. Dnica+Forense+Versi\%C3\%B3n+3+Diciembre+de+2011. pdf/6bf61c74-3789-e215-f1ab-445b2681ad07

Krenzer, U. (2006). Compendio De Métodos Antropológico Forenses (1era. ed., Vol. IV (Estimación de la edadosteológica en subadultos)). Guatemala, Guatemala. Obtenido de http://.cafcaguatemala.org 


\section{Sobre los autores}

\section{Francisco René Fernández Rodas}

Es Cirujano Dentista egresado de la Universidad de San Carlos de Guatemala, Especialización en Odontología Forense con énfasis en Identificación Humana avalado por la Facultad de Ciencias Médicas y Facultad de Odontología de la Universidad de San Carlos de Guatemala, Odontólogo General en Hospital de Rehabilitación del Instituto Guatemalteco de Seguridad Social -IGSS.

\section{Guillermo Rosales Escribá}

Es Profesor del Curso de Morfología y Anatomía Dental por 34 años en la Facultad de Odontología de la Universidad de San Carlos de Guatemala. Investigador sobre Morfología Dentaria desde el Maya Prehispánico hasta el guatemalteco actual. Magister in Artibus en Derechos Humanos. Especialización en Odontología Forense con Énfasis en Identificación Humana. Conferencista nacional e internacional sobre temas relacionados con Antropología Dental. Autor de libros de texto sobre Morfología y Anatomía Dental y sobre técnicas de tallado y dibujo dentario.

\section{Sergio José Zuleta Mejía}

Es Cirujano Dentista egresado de la Facultad de Odontología, USAC $(2,008)$, con Especialización en Odontología Forense con Énfasis en Identificación Humana USAC y autor de la investigación "Odontometría y Variantes Morfológicas del Museo Nacional de Arqueología y Etnología de Guatemala (MUNAE)". Colaboraciones en proyectos de salud en áreas afectadas por desastres naturales en la región de las Verapaces, prestación de servicios profesionales al IGSS, ejercicio de la profesión odontológica en sus clínicas particulares en Alta y Baja Verapaz.

Copyright (c) 2019 Francisco René Fernández Rodas, Guillermo Rosales Escribá y Sergio José Zuleta Mejía

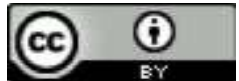

Este texto está protegido por una licencia Creative Commons 4.0.

Usted es libre para Compartir - copiar y redistribuir el material en cualquier medio o formato - y Adaptar eldocumento - remezclar, transformar y crear a partir del material- para cualquier propósito, incluso comercialmente, siempre que cumpla la condición de:

Atribución: Usted debe reconocer el crédito de una obra de manera adecuada, proporcionar un enlace a la licencia,e indicar si se han realizado cambios. Puede hacerlo en cualquier forma razonable, pero no de forma tal que sugieraque tiene el apoyo del licenciante o lo recibe por el uso que hace. 